\title{
Gut microbiota influences Plasmodium falciparum malaria susceptibility
}

\begin{abstract}
Aly Kodio',2, Drissa Coulibaly ${ }^{3}$, Safiatou Doumbo ${ }^{3}$, Salimata Konaté ${ }^{3}$, Abdoulaye Kassoum Koné ${ }^{3}$, Mamadou Lamine TALL 2,4, Ahmed Mohamed Konaté3, Boureima Guindo3, Coralie L'Ollivier 1,2, A. Levasseur 2,4, Fadi Bittar ${ }^{2,4}$, Ogobara K. Doumbo, ${ }^{3, a}$, Didier Raoult ${ }^{2,4}$, Mahamadou Ali Thera ${ }^{3}$ and Stéphane Ranque ${ }^{1,2, *}$
\end{abstract}

1 Aix Marseille Université, Institut de Recherche pour le Développement, Assistance Publique-Hôpitaux de Marseille, Service de Santé des Armées, VITROME : Vecteurs - Infections Tropicales et Méditerranéennes, 1921 Boulevard Jean Moulin, 13005 Marseille, France ; alykodio81@gmail.com (A.K) ; coralie.LOLLIVIER@univamu.fr (C.L).

2 IHU Méditerranée Infection, 19-21 Bd Jean Moulin, 13005 Marseille, France; fadi.BITTAR@univamu.fr (F.B.); anthony.levasseur@univ-amu.fr (A. L) didier.raoult@gmail.com (D.R.) ; laminetall30@gmail.com (M.L.T).

3 Malaria Research and Training Centre-International Center for Excellence in Research (MRTC-ICER), Department of Epidemiology of Parasitic Diseases, Faculty of Medicine and Dentistry, Université des Sciences, des Techniques et des Technologies de Bamako, Point G, BP 1805 Bamako, Mali; okd@icermali.org (O.K.D);

coulibalyd@icermali.org (D.C.); fankone@icermali.org (A.K.K.); sakonate@icermali.org (S.K.); sdoumbo@icerm ali.org (S.D.); ahmedkonate@icermali.org (A.M.K); bguindo@icermali.org (B.G);

mthera@icermali.org (M.A.T.)

4 Aix Marseille Université, Institut de Recherche pour le Développement, Assistance Publique-Hôpitaux de Marseille, Service de Santé des Armées, MEPHI : Microbes, Evolution, Phylogénie et Infection, 19-21 Boulevard Jean Moulin, 13005 Marseille, France

a Deceased

*Correspondence:

Pr Stéphane Ranque

IHU Méditerranée Infection

19 Bd Jean Moulin

F-13005 Marseille

E-mail : stephane.ranque@univ-amu.fr 


\section{Abstract}

The gut microbiota has recently been associated with susceptibility/resistance to malaria in animal models and humans, yet the impact of the gut microbiota on the risk of a malaria attack remains to be assessed. This study aims at assessing the influence of the gut microbiota on malaria attacks and Plasmodium parasitæmia in children living in a malaria-endemic area in Mali. Three hundred healthy children were included in a 16-months cohort study in Bandiagara. Their gut bacteria and fungi community structures were characterised via $16 \mathrm{~S}$ and ITS metabarcoding from stool samples collected at inclusion. Clinician team monitored the occurrence of malaria attacks. Asymptomatic carriage of Plasmodium was assessed by qPCR.

Over the 16-month period, 107 (36\%) children experienced at least one occurrence of malaria attacks, and $82(27 \%)$ at least one asymptomatic Plasmodium parasitæmia episode. A higher gut bacteria richness was independently associated with susceptibility to asymptomatic parasitæmia episodes and malaria attacks; while the Shannon H diversity and Chao-1 richness index of gut fungi community structure was relatively homogeneous in children who were and were not infected with $P$. falciparum. Using a linear discriminant effect size analysis of operational taxonomic units assigned to the species level, 17 bacteria, including Clostridiaceae, Eubacteriaceae, Senegalimassilia sp., Atopobiaceae and Lachnosipraceae, and seven fungi, including Dioszegia fristigensis, Ogataea polymorpha and Cutaneotrichosporon cyanovorans, were associated with susceptibility; whereas eight bacteria, including, Bifidobacterium spp., Weissela confusa and Peptostreptococcacea, and 3 fungi, Malassezia sp., Niesslia exosporoides, and Didymocrea leucaenae, were associated with resistance to malaria. Moreover, 15 bacteria, including Coproccus eutactus, Terrisporobacter petrolearius, Klebsiella pneumoniae and Ruminococcaceae, and 13 fungi, including Wallemia mellicola, were associated with susceptibility, whereas 19 bacteria, including Bifidobacterium spp., Bacteroides fragilis, Peptostreptococcacea, and Lactobacillus ruminis, and three fungi, including Cryptococcus neoformans, were associated with resistance to asymptomatic Plasmodium parasitæmia episodes. Further studies are needed to confirm these findings that point the way towards strategies aiming to reduce the risk of malaria by modulating gut microbiota components in atrisk populations.

Keywords: Gut microbiota, Mycobiota, Bacteria, Fungi, Malaria, Plasmodium falciparum, 16S metagenomics, ITS metagenomics, Children, Cohort, Mali, Dogon country. 


\section{Introduction}

Malaria is caused by a protozoan parasite, Plasmodium spp., which are transmitted to humans through the infesting bite of a female Anopheles mosquito. The highest malarial burden is clustered in Africa and the area south of the Sahara makes up $98 \%$ of the 228 million cases worldwide each year. Plasmodium falciparum malaria is the most life-threating parasitic disease worldwide, despite the implementation of multiple control strategies. It causes 405,000 deaths or more each year and children under the age of five are the most vulnerable population, making up $67 \%(272,000)$ of these deaths [1].

Malaria parasite carriage can progress as an asymptomatic infection, as uncomplicated malaria with symptoms as fever, headache, and chills, or as severe malaria including high parasitæmia, severe anaemia, respiratory distress, and cerebral malaria (altered consciousness and seizures) $[2,3]$. However, factors have been described to be associated with malarial resistance, including ethnic and genetic factors, such as sickle cell traits (AS, C), Duffy negative blood group, HLA group, and polymorphisms in immune response genes [3-8]. In areas of intense malaria transmission, Young children are more susceptible to malaria due to the loss of maternal antibody protection from 9 months. They present a high malaria morbidity and mortality. Older children, as a result of repeated malaria attacks, acquire a non-sterilising premunition that reduces the risk of severe malaria and associated case fatality $[9,10]$. The immune response to Plasmodium infection is partially known and the occurrence of malaria is variable from one individual to another and in the same individual $[11,12]$. This poses the difficulty of developing an effective malaria vaccine which is also linked to the complexity of the biology of Plasmodium and the arsenal that it has developed over the years to adapt to the immunity of its hosts $[13,14]$.

Recently, the gut bacterial community has been pointed out as a protective factor against Plasmodium infection in humans. In a recent study, anti- $\alpha$-gal antibodies were associated with protection against Plasmodium infection in humans and in the mouse model. Anti- $\alpha$-gal antibodies, induced by the pathobiont Escherichia coli O86:B7 in the mouse gut, are cytotoxic to antigens on the surface of Plasmodium sporozoites, thus protecting mice from the transmission of Plasmodium infection by mosquitoes. The same study reported an association between anti- $\alpha$-gal IgM levels and protection against malaria infection [15]. Also, natural anti$\alpha-\mathrm{Gal} \operatorname{IgG} 3$ and $\mathrm{IgG} 4$ antibody levels were raised in children who had experienced no malaria attack within one year of follow-up, indicating evidence of protection against malaria infection in Mozambican children [16]. Beyond the transmission of Plasmodium infection, the gut 
microbiota has been associated with severe malaria infection. Lactobacillus and Bifidobacterium species in the gut have shown to play a protective role against Plasmodium infection by reducing the parasite load and attenuating the severity of malaria in mice [2]. Severe malaria infections alter the functional capacity of the microbiota, improving bacterial motility and amino acid metabolism in mice with a high parasite load compared to a mild infection [17]. Severe malaria infection can be modulated by the gut microbiota in genetically diverse mice and pregnant animals. The abundance of Akkermansia muciniphila, Allobaculum, Lactobacillus and S24-7 has also shown to be negatively correlated with parasite load[18]. Also, severe malaria infection in pregnant mice, which is a function of the composition of the intestinal microbiota, significantly influences foetal and postnatal outcomes [18]. Notably, the bacterial of the microbiota was associated with the risk of asymptomatic Plasmodium parasitæmia occurrence and not of malaria attack [19]. However, no studies have yet established that the bacterial microbiota contributes towards protecting against malaria in humans, and the influence of the fungal microbiota on malaria has not yet been investigated.

This study aimed to assess whether both bacteria and fungi communities in the gut were associated to the susceptibility/resistance against asymptomatic Plasmodium infection and malaria attacks in children living in a malaria-endemic area, Bandiagara (Mali).

\section{Results}

\subsection{Children's baseline characteristics}

Three hundred (300) children were included in the cohort study; the median age of the cohort was 8 IQR [7; 8], respectively, from which the ages were stratified. Stools were able to collect from 296/300 children. Microbiota analysis of the faeces of the 296 children was performed. From these apparently healthy 296 children, asymptomatic Plasmodium sp. parasitæmia was detected by PCR in 35 (12\%). Asymptomatic baseline P. falciparum parasitæmia was $11 \%$ (33/296)) or P. ovale $0.6 \%$ (2/296). From eukaryotic enteric pathogens protists, including Blastocystis sp. (49.7\%) and Giardia lamblia (29\%) were the more prevalent, whereas helminths were rarely detected, i.e., Trichuris trichiura was present in only $1 \%$ and Schistosoma mansoni in $0.3 \%$ (Table 1). The number of malaria attacks totalled 107 and the number of children carriage of asymptomatic Plasmodium parasitæmia was 82 during the 16 months of follow-up. Age group 0-4-year-old had fewer malaria attacks and asymptomatic Plasmodium parasitæmia compared to the older age group (Table S1). 
Table 1. Baseline characteristics of the children included in the cohort study, by age group

\begin{tabular}{|c|c|c|c|c|c|}
\hline \multirow{2}{*}{ Characteristics } & \multicolumn{4}{|c|}{ Age groups } & \multirow[t]{2}{*}{ Total } \\
\hline & $6 \mathrm{mo}-4 \mathrm{y}$ & $5-8$ y & 9-11 y & $12-15 y$ & \\
\hline $\mathbf{N}(\%)$ & $78(26 \%)$ & $90(30 \%)$ & $69(23 \%)$ & $63(21 \%)$ & $(n=300)$ \\
\hline Male & $37(47 \%)$ & $48(53 \%)$ & $33(47 \%)$ & $28(44 \%)$ & $146(47 \%)$ \\
\hline \multicolumn{6}{|l|}{ Ethnicity } \\
\hline Dogon & $55(70 \%)$ & $61(68 \%)$ & $46(67 \%)$ & $38(60 \%)$ & $200(66 \%)$ \\
\hline Songhai & $7(9 \%)$ & $6(6.7 \%)$ & $7(10 \%)$ & $8(13 \%)$ & $28(9 \%)$ \\
\hline Fulani & $2(2.6 \%)$ & $5(5.6 \%)$ & $4(5.8 \%)$ & $6(9 \%)$ & $17(6 \%)$ \\
\hline Others & $14(18 \%)$ & $18(20 \%)$ & $12(17 \%)$ & $11(17 \%)$ & $55(18 \%)$ \\
\hline Malaria attack & 0 & 0 & 0 & 0 & 0 \\
\hline Subjected to qPCR (N) & 77 & 90 & 67 & 62 & 296 \\
\hline Plasmodium sp. & $2(2.6 \%)$ & $8(9 \%)$ & $8(12 \%)$ & $17(27 \%)$ & $35(12 \%)$ \\
\hline$P$. falciparum & $2(2.6 \%)$ & $8(9 \%)$ & $7(10 \%)$ & $16(25 \%)$ & $33(11 \%)$ \\
\hline P. ovale & 0 & $0 / 90$ & $0 / 67$ & $2(3.2 \%)$ & $2(0.6 \%)$ \\
\hline Blastocystis sp. & $26(34 \%)$ & $49(54 \%)$ & $41(61 \%)$ & $31(50 \%)$ & $\begin{array}{l}147 \\
(49.7 \%)\end{array}$ \\
\hline Giardia lamblia & $20(26 \%)$ & $34(38 \%)$ & $21(31 \%)$ & $12(19 \%)$ & $87(29 \%)$ \\
\hline Dientamoeba fragilis & 0 & $1(1 \%)$ & $2(3 \%)$ & 0 & $3(1 \%)$ \\
\hline Balantidium coli & 0 & $1(1 \%)$ & 0 & 0 & $1(0.3 \%)$ \\
\hline $\begin{array}{l}\text { Encephalitozoon } \\
\text { intestinalis }\end{array}$ & 0 & 0 & 0 & 0 & 0 \\
\hline Cyclospora cayetanensis & 0 & $1(1 \%)$ & 0 & 0 & 0 \\
\hline Cryptosporidium parvum & 0 & 0 & 0 & 0 & 0 \\
\hline Entamoeba histolytica & 0 & $1(1 \%)$ & $1(1.4 \%)$ & $2(3.2 \%)$ & $4(1.3 \%)$ \\
\hline Isospora belli & 0 & 0 & $1(1.4 \%)$ & 0 & $1(0.3 \%)$ \\
\hline Enterocytozoon bieneusi & $7(9 \%)$ & 0 & 0 & $3(4.8 \%)$ & $10(3.4 \%)$ \\
\hline Ancylostoma duodenale & 0 & 0 & 0 & 0 & 0 \\
\hline Ascaris lumbricoides & 0 & 0 & 0 & 0 & 0 \\
\hline Hymenolepis diminuta & 0 & 0 & 0 & 0 & 0 \\
\hline Necator americanus & 0 & 0 & 0 & 0 & 0 \\
\hline Schistosoma mansoni & 0 & 0 & 0 & $1(1.6 \%)$ & $1(0.3 \%)$ \\
\hline Strongyloides stercoralis & 0 & 0 & 0 & 0 & 0 \\
\hline Taenia solium & 0 & 0 & 0 & 0 & 0 \\
\hline Taenia saginata & 0 & 0 & 0 & 0 & 0 \\
\hline Trichuris trichiura & 0 & $1(1 \%)$ & 0 & $2(3.2 \%)$ & $3(1 \%)$ \\
\hline Enterobius vermicularis & 0 & 0 & 0 & 0 & 0 \\
\hline
\end{tabular}




\subsection{Gut bacteria 16 S metabarcoding}

From the 296 stool samples, 24,913,960 sequences were generated for taxonomic assignment. Sequences not classified at the species level and the other classes were indicated "unassigned" for taxonomy. Some classified sequences were also labelled as IHU_Bacteria (bacteria from the IHU database) with distinct numbers. The analysis of Operational Taxonomic Units (OTUs) in the children's gut bacterial communities showed a relatively evenly distributed frequency of the major phyla, Firmicutes (12\%), Bacteroides (12\%), Actinobacteria (12\%) and Proteobacteria (12\%), and a relative higher abundance of Firmicutes (47\%) compared to other phyla, including Bacteroides (8\%), Actinobacteria (8\%) and Proteobacteria (6\%) (Figure 1A, 1B).

(A)

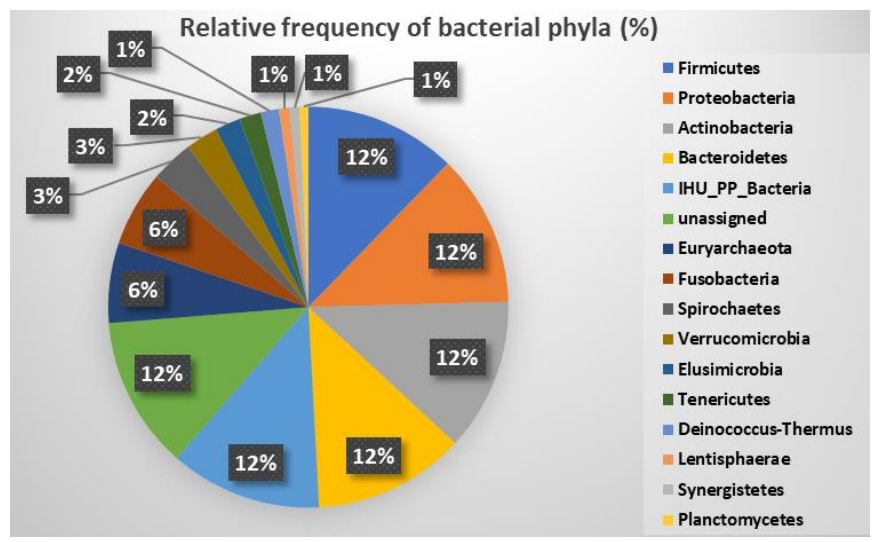

(C)

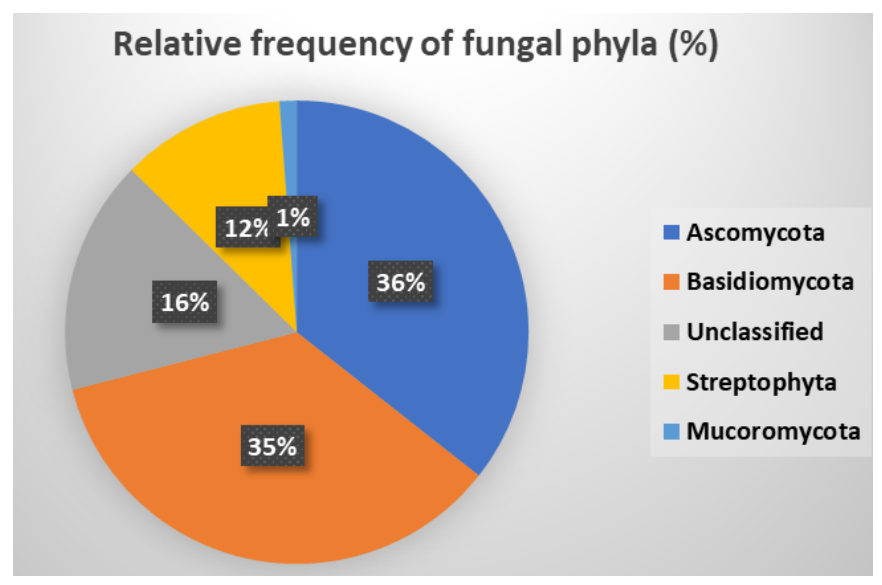

(B)

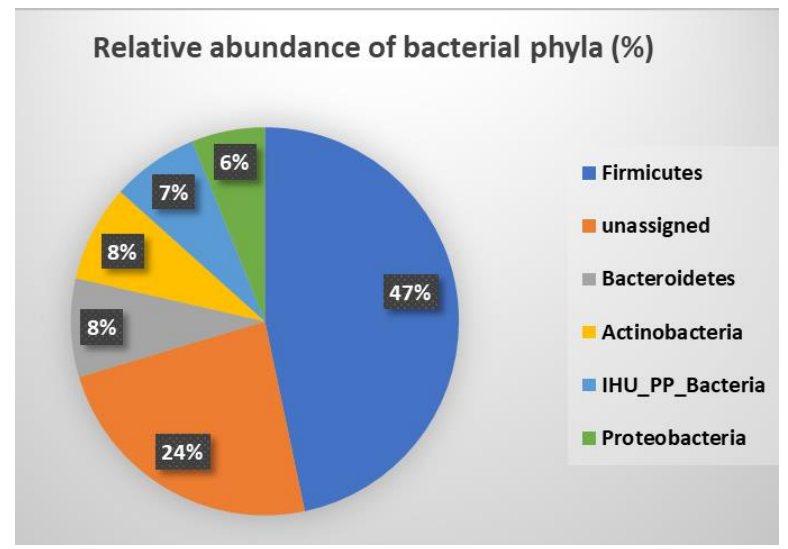

(D)

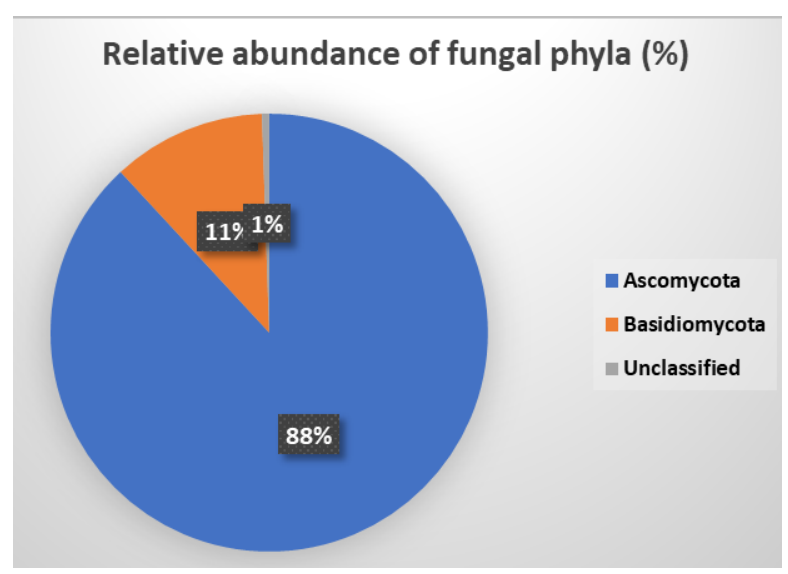

Figure 1. Relative frequency (A) and abundance (B) of the major gut bacterial phyla, and relative frequency (C) and abundance (D) of the major gut fungal phyla, characterised via 16S or ITS metabarcoding. 


\subsection{Gut fungi ITS metabarcoding}

The ITS1 region analysis yielded 647,816 reads and 532 single OTUs; the ITS2 region yielded 1,975,320 reads and 479 single OTUs. Of the 1011 detected fungal taxa, 53 were identified in ITS1 reads and 479 in both ITS1 and ITS2 reads. The ITS1 and ITS2 OTU tables were combined for all further analyses.

The analysis of Operational Taxonomic Units (OTUs) in the children's gut fungal communities showed that relatively evenly distributed frequency of the two major phyla, Ascomycota (35.5\%) and Basidiomycota (35.3\%). It should be noted that Plantae Streptophyta accounted for $11 \%$ of the OTUs (mostly amplified with the ITS2 barcode) and $16.3 \%$ remained unclassified. The most abundant phyla were Ascomycota (88\%) and Basidiomycota (11\%) (Figure 1C, 1D). Because gut fungi are less complex and relatively less known than bacterial communities, we further analysed the distribution of the fungal taxa according to the children's age groups. All fungi phyla were detected in children under the age of five; each phylum frequency was relatively higher in the 5 to 10-year-old group (Figure 2A). Fungal phyla abundance relatively peaked in children under the age of 5 to 10-year-old and then decreased with age (Figure 2B). 
(A)

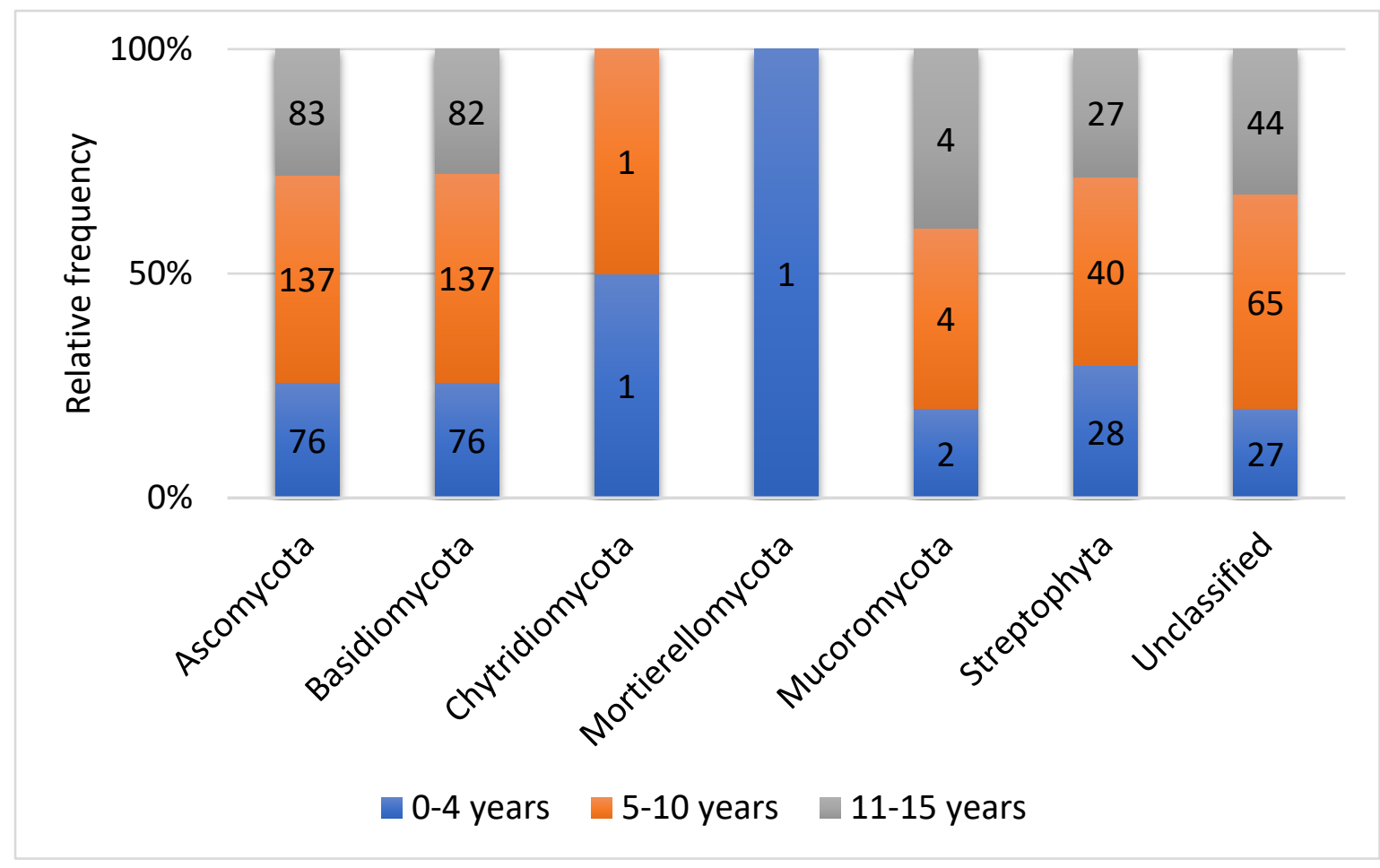

(B)

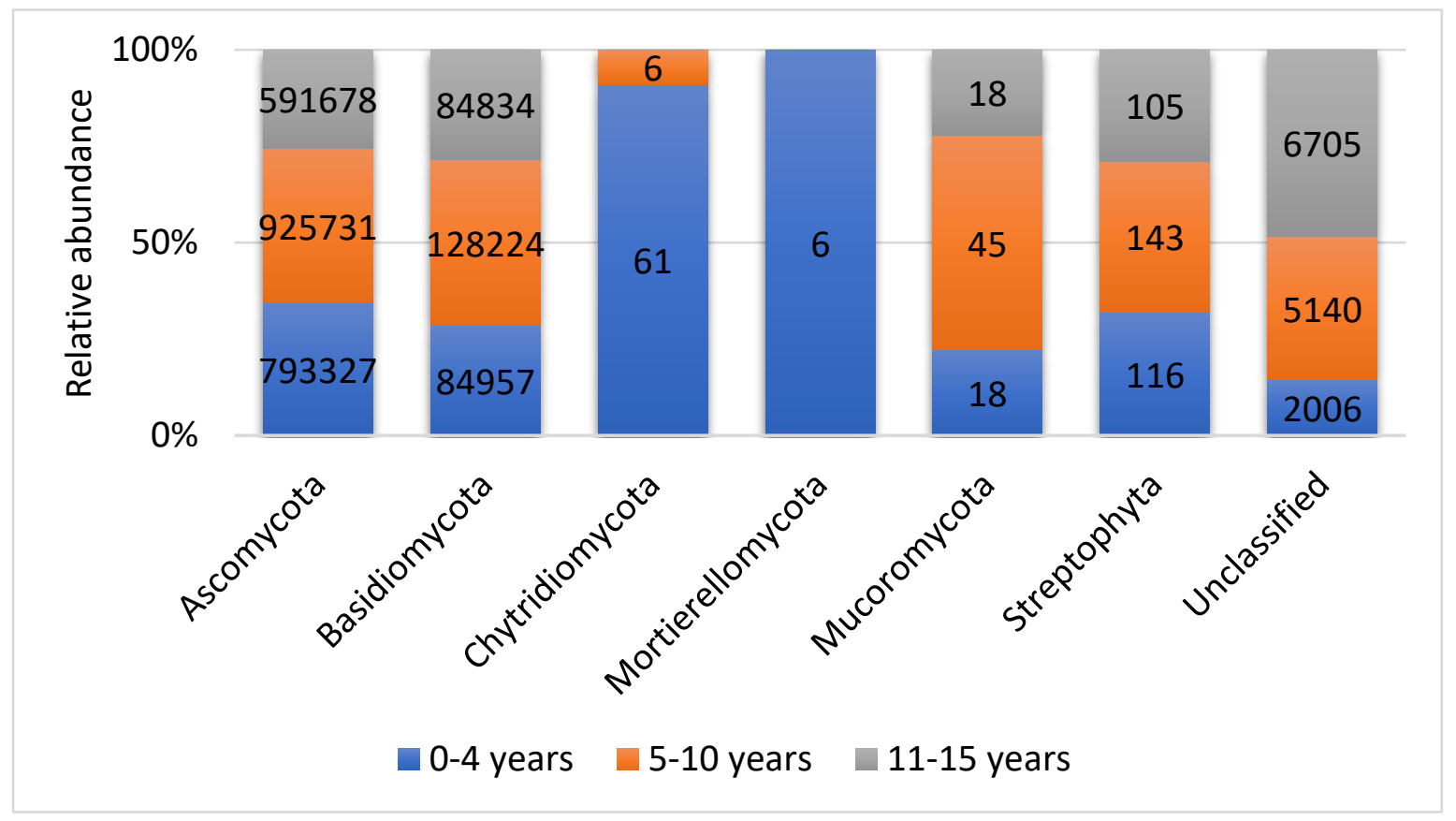

Figure 2. Distribution of the relative frequency A), and abundance B) of gut fungi phyla metabarcoding reads according to age. (Figures inside the bars are the mean abundance and number of the phylum for each group). 


\subsection{Malaria disease patterns during the 16 months of follow-up}

Thirty-five (35) children with asymptomatic Plasmodium parasitæmia at baseline were excluded from these analyses and the median age of the remaining 258 children was seven years (range=0-15, IQR [4-10.25]). A natural logarithmic transformation of age (in years) was best suited to the logistical regression model of both malaria attacks and asymptomatic Plasmodium parasitæmia risks. The best-fitting multivariate logistical regression analysis model found that age $(\mathrm{OR}=1.66(1.11-2.48), \mathrm{p}=0.014)$, and OTU richness $(\mathrm{OR}=1(1.000-1.002), \mathrm{p}=0.004)$, were independently statistically significantly associated with the risk of developing at least one attack (Table 2).

Table 2. Logistic regression analysis of the association between age and gut bacterial and fungal community structure with the risk of malaria attack.

\begin{tabular}{|c|c|c|c|c|}
\hline \multirow{2}{*}{ Variables } & \multicolumn{2}{|c|}{ Univariate analysis } & \multicolumn{2}{c|}{ Multivariate analysis } \\
\cline { 2 - 5 } & Odd Ratio (95\% CI) & P value & Odd Ratio (95\% CI) & P value \\
\hline Age & $6.32(1.57-25.35)$ & 0.009 & $1.66(1.11-2.48)$ & 0.014 \\
\hline Bacteria community & $(\mathrm{n}=107 / 300,35.67 \%)$ & & & \\
\hline OTU Richness & $1.001(1-1.002)$ & 0.001 & $1(1-1.002)$ & 0.004 \\
\hline Chao-1 index & $2.04(1.05-3.94)$ & 0.035 & $1(1-1.002)$ & 0.432 \\
\hline Shannon index & $1.51(0.81-2.83)$ & 0.197 & - & - \\
\hline Simpson index & $0.66(0-7569.24)$ & 0.929 & - & - \\
\hline Fungi community & $(\mathrm{n}=107 / 300,35.67 \%)$ & & - & - \\
\hline OTU Richness & $1(0.994-1)$ & 0.647 & - & - \\
\hline Chao-1 index & $0.998(0.989-1.007)$ & 0.633 & - & - \\
\hline Shannon index & $0.83(0.40-1.76)$ & 0.623 & - & - \\
\hline
\end{tabular}

Best-fitting transformation model used natural logarithm function of: Age; Chao indices; Richness; and Simpson indices; and power function of Shannon indices.

Similarly, age $(\mathrm{OR}=2.10(1.32-3.35), \mathrm{p}=0.002)$, and the diversity indices, Shannon $\mathrm{H}(\mathrm{OR}=5.34$ (1.05-27.67), $\mathrm{p}=0.043)$ and the Simpson D natural logarithm $(\mathrm{OR}=0.001(0-0.026), \mathrm{p}=0.024)$, were independently statistically significantly associated with the risk of developing at least one asymptomatic Plasmodium parasitæmia episode (Table S2). 
Both age and OTU richness were significantly associated with both malaria attack and asymptomatic Plasmodium parasitæmia in the univariate analysis. The survival analysis showed that being over the age of four was associated with an increased risk of both malaria attack $(\mathrm{p}=0.015)$ and asymptomatic Plasmodium parasitæmia $\left(\mathrm{p}<10^{-3}\right)($ Figure S1A, S1B). The median OTU richness was 1,106 (112-2,551) and the median Chao-1 index was 1,303 (428$3,979)$. To estimate the risk of malarial infection, the cohort of 258 children were divided in two groups either lesser than or equal to and above the median of the richness indices (observed OTU richness and Chao-1). Cox regression analysis showed that a relatively low OTU richness was associated with a significantly lower risk of malaria attack than with high OTU richness in children (Hazard Ratio $=0.59$; 95\%CI $[0.42-0.84], \mathrm{p}=0.0031$ ). Similarly, children with a relatively low OTU richness showed a lower risk of asymptomatic Plasmodium parasitæmia episodes than children with higher OTU richness (Hazard Ratio=0.60; 95\%CI [0.44 - 0.81], $\mathrm{p}=0.0009$ ) (Figure S2A, S2B). To assess whether age could be a confounding factor, the same analysis was conducted in children aged 0-4 years old or aged 5 years old and above. Children aged 0-4 years old with an OTU richness below the median value (1109; IQR [680 - 1307]) showed a lower risk of both malaria attack and asymptomatic Plasmodium parasitæmia episodes than children with an OTU richness above the median value ( $\mathrm{p}=0.03)$ (Figure S3). This effect was even enhanced in children aged 5-15 years, where those with an OTU richness below the median value (1109; IQR [680 - 1307]) showed a lower risk of both malaria attack and asymptomatic Plasmodium parasitæmia episodes than those with an OTU richness above the median value $(\mathrm{p}<0.0001)$ (Figure S4).

\subsection{Malaria in the Dogon and Fulani ethnic groups}

Regarding these two sympatric ethnic groups in Bandiagara, more Dogon ( $n=200)$ than Fulani $(n=17)$ children were included in this study. The median age of the Dogon $(7$ [4-15]) and Fulani $(10$ [1-14]) children was homogeneous $(\mathrm{p}=0.12)$. Overall, $74(37 \%)$ of the Dogon and four $(24 \%)$ of the Fulani children $(\mathrm{p}=0.306)$ developed at least one malaria attack and $53(27 \%)$ of the Dogon and seven $(41 \%)$ and of Fulani children $(\mathrm{p}=0.257)$ developed at least one asymptomatic Plasmodium parasitæmia episode during the 16-month follow-up period. Because of the relatively small number of Fulani enrolled into the study, the impact of the gut bacterial and fungal communities in these ethnic groups was not investigated further.

\subsection{Gut bacterial community structure associated with malarial risk}


The association of age, sex, eukaryotic enteric pathogens, and gut bacterial and fungal community structures with either the risk of a malaria attack or asymptomatic Plasmodium parasitæmia, were assessed using an unconditional logistic regression analysis. Univariate analysis showed that age and gut bacteria richness indices (Table 2) were significantly associated with malaria attacks $(\mathrm{p}<0.05)$. In the multivariate analysis, age and OTU richness were statistically significantly associated with malaria attacks OR=2.62, (95\% CI [1.24-5.54] and $\mathrm{OR}=1.66(95 \% \mathrm{CI}[1.11-2.49])$ respectively (Table 2). Similarly, age (OR=2.27, 95\%CI [1.43-3.62]) and OTU richness $(\mathrm{OR}=2.44$, 95\%CI [1.163-5.123]) were statistically significantly associated with asymptomatic Plasmodium parasitæmia.

The gut bacterial community structure of children who developed at least one malaria attack were compared with those who did not, and that of those who developed at least one asymptomatic Plasmodium parasitæmia episode was compared with those who did not. At the phylum level, the relative abundance of the main bacterial phyla were Firmicutes $(53.95 \%)$, Proteobacteria (53.84\%), Actinobacteria (52.9\%), and Bacteroides (49.54\%) in the children who developed at least one malaria attack compared to those who did not (Figure S5); and Proteobacteria (55.3\%), Firmicutes (52.83\%), Actinobacteria (49.97\%), and Bacteroides $(49.52 \%)$ in children who developed at least one asymptomatic Plasmodium parasitæmia episode compared to those who did not (Figure S6). The impact of the gut bacteria community structure on malarial risk was further assessed via Principal Coordinates Analysis (PCoA) and tested using PERMANOVA, based on the Bray-Curtis similarity measure. It significantly differed in the children who developed at least one malaria attacks compared to those who did not $(\mathrm{p}=0.005)$ (Figure 3a) and differed in those who developed at least one asymptomatic Plasmodium parasitæmia episode compared to those who did not $(\mathrm{p}=0.012)$ (Figure 3b). 


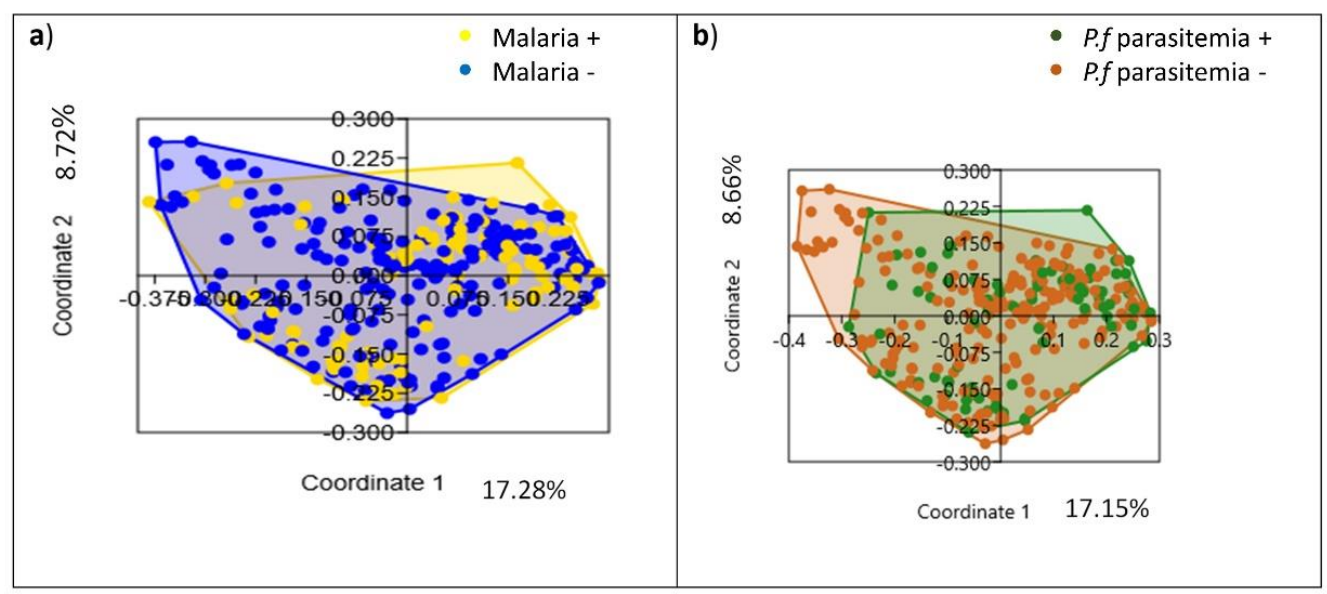

Figure 3. Gut bacterial community structure and malaria risk. Principal Coordinates Analysis (PCoA) of the gut bacterial community a) in children who experienced (yellow dots), or did not experience (blue dots), at least one malaria attack within 16 months of follow-up (Permanova test, $\mathrm{p}=0.0054)$; $\mathrm{b}$ ) in children who developed (green dots), or did not develop (pink dots), at least one asymptomatic Plasmodium parasitaemia episode within 16 months of follow-up (Permanova test, $\mathrm{p}=0.012$ ).

Bacterial OTU richness (Chao-1 index and observed OTU richness) and diversity in Shannon $\mathrm{H}$ and Simpson D indices were evaluated according to malaria status and age (Table S3). The diversity indices were homogeneously distributed in each of the groups of children (Table S3). In contrast, the Chao-1 index (median 1,457 [1,352-1,561]) in the children who developed at least one malaria attack was significantly higher than in those who did not $(1,330[1,257-$ 1,402]) $(\mathrm{p}=0.036)$. Also, the median bacterial OTU richness observed $(1,233$ [1,152-1,313]) in the children who developed at least one malarial attack was statistically significantly higher $(\mathrm{p}=0.001)$ than that observed in those who did not $(1,076[1,022-1,129])$ (Figure 4; Table S4). 
A)

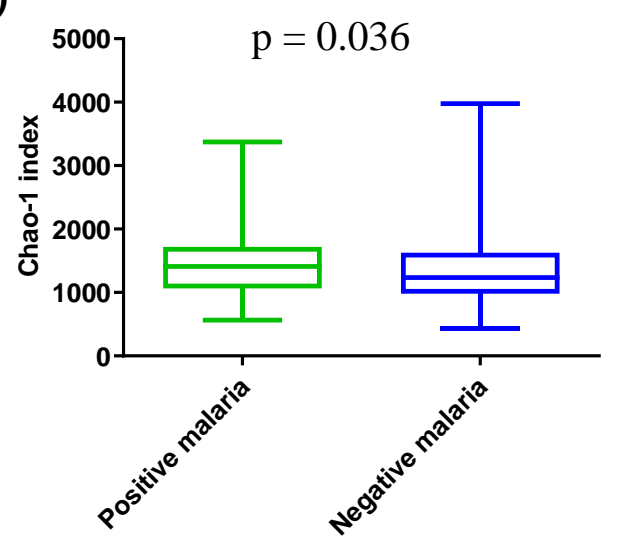

C)

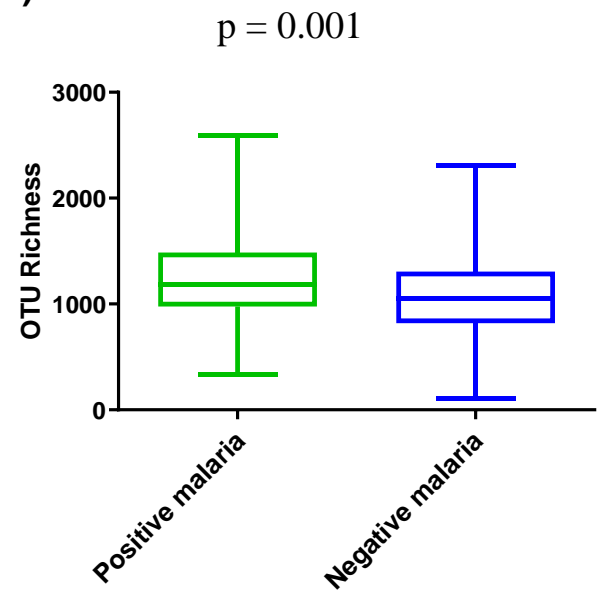

B)

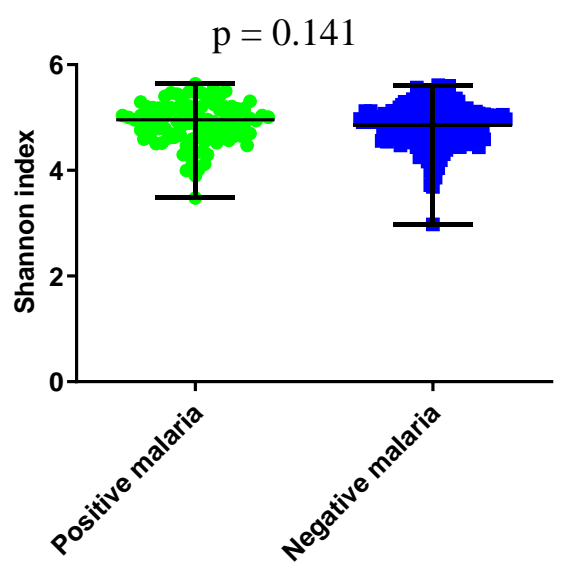

D)

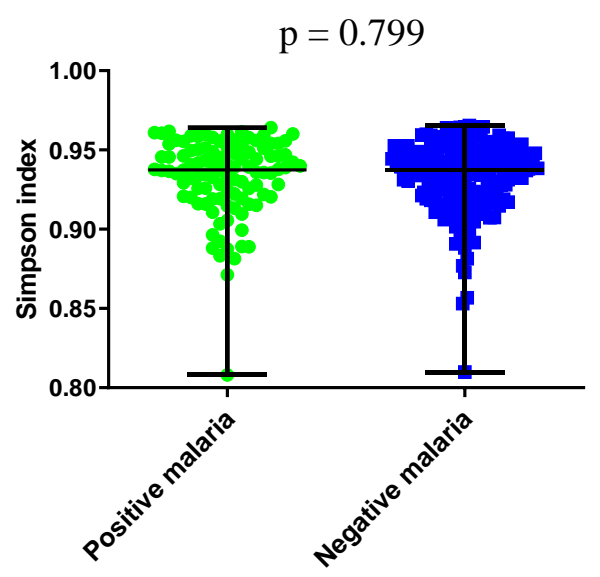

Figure 4. Richness and diversity indices of the gut bacterial community in children who experienced or did not experience at least one malaria attack within 16 months of follow-up. Scatter dot plot and Box-Whisker's graph shows Median, Min and Max of children who experienced at least one malaria attack (green) compared to control (blue) A) for chao-1 index $(\mathrm{p}=0.036)$; B) for Shannon index $(\mathrm{p}=0.141)$; C) for OTU Richness $(\mathrm{p}=0.001)$; $\mathrm{D})$ for Simpson index $(\mathrm{p}=0.799)$.

We found that the median bacterial OTU richness observed in the children who developed at least one asymptomatic Plasmodium parasitæmia episode (1,217 [1,130-1,305]) was higher $(\mathrm{p}=0.02)$ than in those who did not $(1,099$ [1,046-, 1,151]) (Figure 5; Table S5). 
a)
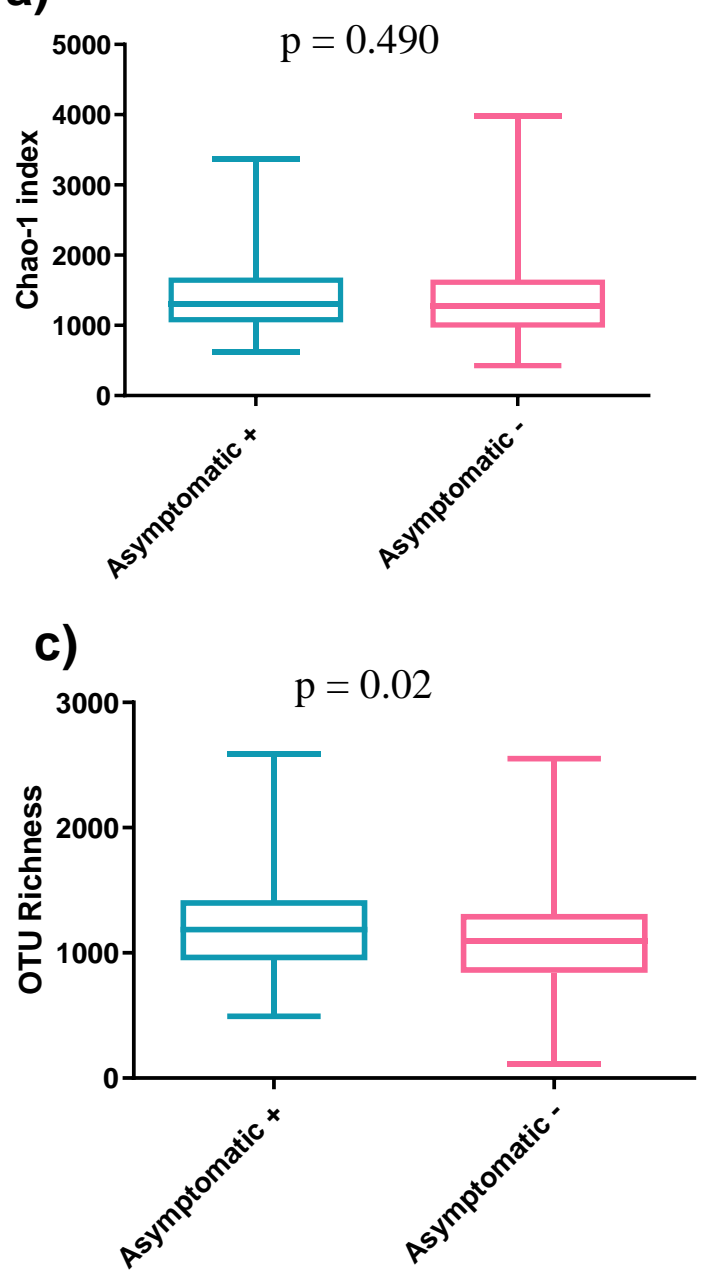

b)

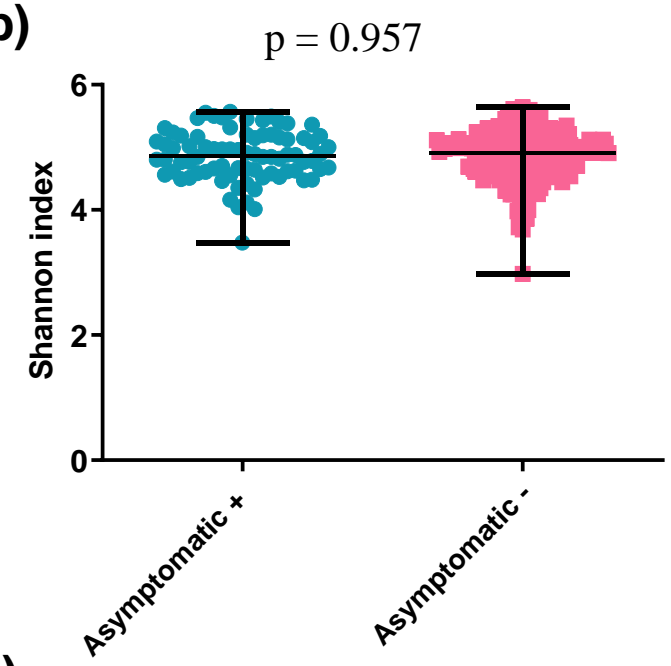

d)

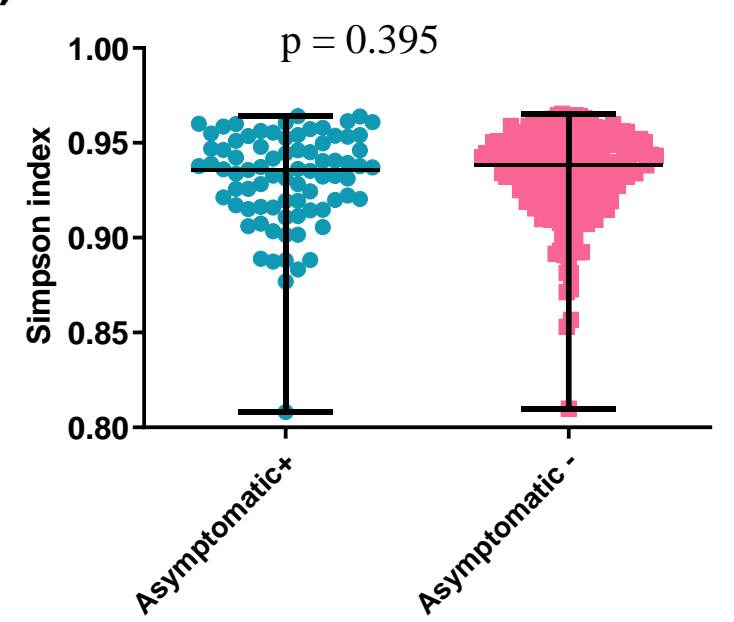

Figure 5. Richness and diversity indices of gut bacterial community of children who developed or did not develop at least one asymptomatic Plasmodium parasitaemia episode within 16 months of follow-up. Median and range of chao-1 index $(\mathrm{p}=0.049) \mathrm{a})$; of Shannon index $(\mathrm{p}=0.957) \mathrm{b})$; for OTU Richness $(\mathrm{p}=0.02) \mathrm{c})$ and Simpson index $(\mathrm{p}=0.395) \mathrm{d})$ of children who developed at least one asymptomatic Plasmodium parasitaemia episode (light blue) compared to control (pink) for Scatter dot plot, Box and Whiskers graph.

The presence of significantly differentially distributed bacterial taxa in children who developed at least one malaria attack and those who did no was assessed using a linear size effect discriminant analysis (LDA LEfSe) at various taxonomical levels (Figures 6a-d). At the Order level, Actinomycetales, Selenomonadales, Aeromonadales, Oceanospirillales, and Acidaminococcales were more abundant in the children who developed at least one malaria attack, whereas Bifidobacteriales were more abundant in those who did not. At the Family level, mainly Coriobacteriaceae, Eubacteriaceae, Actinomycetaceae, and Staphylococacceaea were 
more abundant in the children who developed at least one malaria attack, whereas Peptostreptococcaceae, Clostridiaceae, Psychromonadaceae, and Burkholderiaceae were more abundant in those who did not. At the genus level, IHU- Clostridiacea, IHU-Lachnospiraceae, Intestinibacter, IHU-Actinomycetaceae, Slackia, Gordonibacter, IHU-Selenomonadaceae and Blastococcus were more abundant in the children who developed at least one malaria attack, whereas Bifidobacterium, Weissella, and Veillonella were more abundant among those who did not. At the species level, Clostridium sp. were most abundant in the children who developed at least one malaria attack, whereas Bifidobacterium faecale, Bifidobacterium longum subsp. suillum, Weissela confusa, Peptostreptococcaceae sp., Dorea longicatena, Dorea timonensis, and Streptococcus timonensis, were more abundant in those who did not (Figures 6a-d). Regarding the effect of age on bacterial community structure: in 0-4 years old children who developed at least one malaria attack, from 68 species, Terrisporobacter_petrolearius, UHI_PS_93_Ruminococcaceae_2772 were more abundant, whereas in children who did not 17 species, including Streptococcus salivarius subsp. salivarius and Bifidobacterium faecale were more abundant (Figure S7); in 5-15 years old children who developed at least one malaria attack, 14 species, including Streptococcus lutetiensis and IHU_PS_95_Gemmiger_1543, were more abundant, whereas 9 species, including Prevotella copri and IHU_PS_96_Roseburia_2390, were more abundant in children who did not (Figure S8).

Regarding the children who developed at least one asymptomatic Plasmodium parasitæmia episode, the LDA LEfSe test showed a higher abundance of the Proteobacteria phylum, whereas Bacilli and Negativicutes were more abundant in children who developed no parasitæmia. At the class level, Gammaproteobacteria and clostridia were more abundant in children who developed at least one asymptomatic episode of Plasmodium parasitæmia and Bacilli and Negativicutes were more abundant in children who did not develop at least one asymptomatic Plasmodium parasitæmia. At the order level, children who developed at least one asymptomatic episode of Plasmodium parasitæmia had a greater abundance of Clostridiales and Enterobacteriales, while children without an asymptomatic episode of Plasmodium parasitæmia showed a greater abundance of Lactobacilli, Bifidobacteriales, Eggerthellales and Veillonellales. The same trends were observed at the Family level: Clostridiaceae, Enterobacteriaceae, Archandiaceae were more abundant in the children who developed at least one asymptomatic Plasmodium parasitæmia episode, whereas Lactobacillaceae, Bifidobacteriaceae, Eggerthellaceae, and Veillonellaceae were more abundant in those who did not. At the genus level, Clostridium, Klebsiella, and IHU-Enterobacteriaceae were more 
abundant in the children who developed at least one asymptomatic Plasmodium parasitæmia episode, whereas Bifidobacterium, Bacteroides, Lactobacillus, Dialister, Rothia, and Veillonella were more abundant in those who did not. At species level, Clostridium sp., Klebsiella sp., Ruminococcus sp., Ramboutsia sp. were more abundant in children who developed at least one asymptomatic Plasmodium parasitæmia episode, whereas Bifidobacterium sp., Bacteroides fragilis, and Lactobacillus ruminis were more abundant in those who did not (Figures 7A-D).

Regarding the effect of age on bacterial community structure; children from 0-4 years old who developed at least one asymptomatic episode of Plasmodium parasitæmia, Romboutsia timonensis and Ruminococcus bromii were more abundant among 107 species, whereas in children who did not 5 species, Collinsella aerofaciens and Bifidobacterium longum subsp. longum were more abundant (Figure S9); in 5-15 years old group who developed at least one asymptomatic episode of Plasmodium parasitæmia, 6 species, including pneumoniae subsp. pneumoniae and IHU_PS_96_Ruminococcus_395, were more abundant, whereas 10 species, including Bacteroides fragilis and IHU_PS_96_Eubacterium_436, were more abundant in children who did not (Figure S10). 


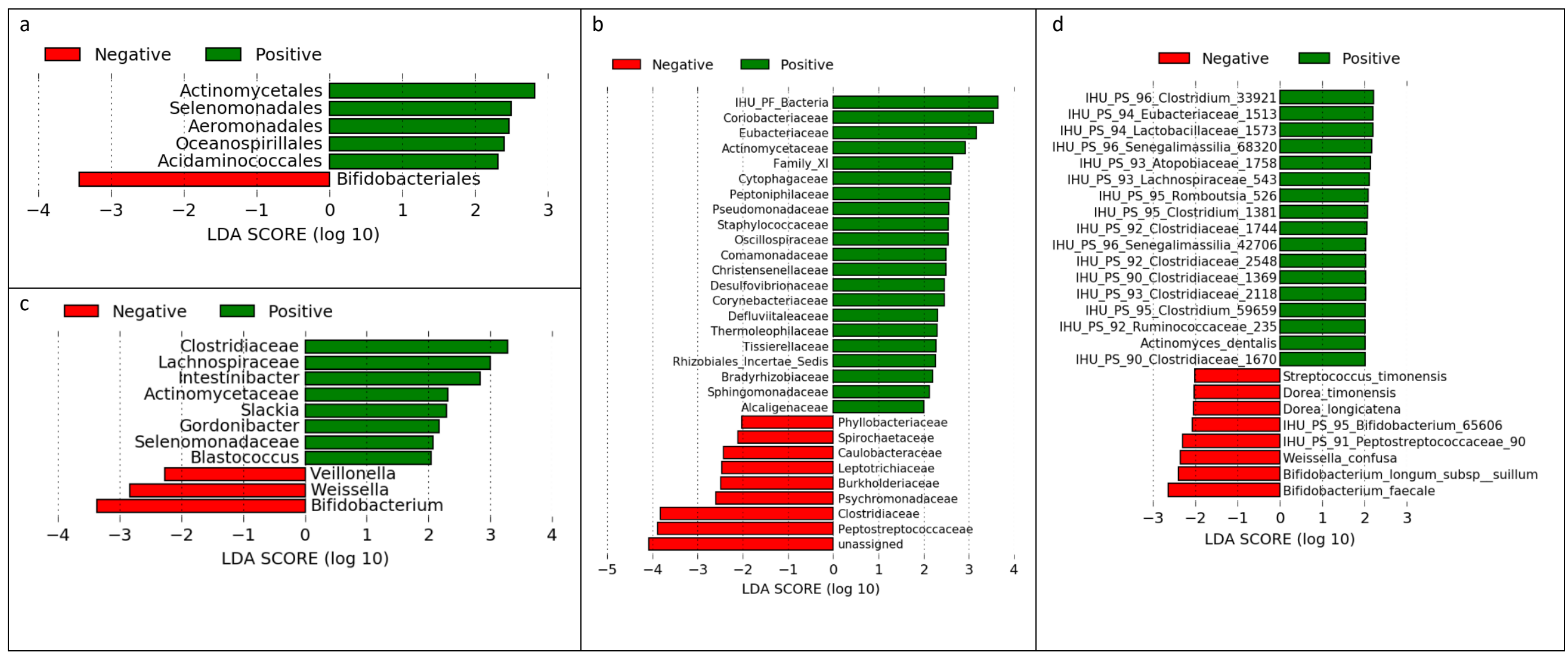

Figure 6. Linear size effect discriminant analysis (LDA LEfSe) of the gut bacterial community structure at, a) Order, b) Family, c) Genus and d) species levels, between children who experienced (green), or did not experience (red), at least one malaria attack within 16 months of follow-up. Horizontal bars represent the effect size for each taxon. The length of the bar represents the $\log 10$ transformed LDA score, indicated by vertical dotted lines. 


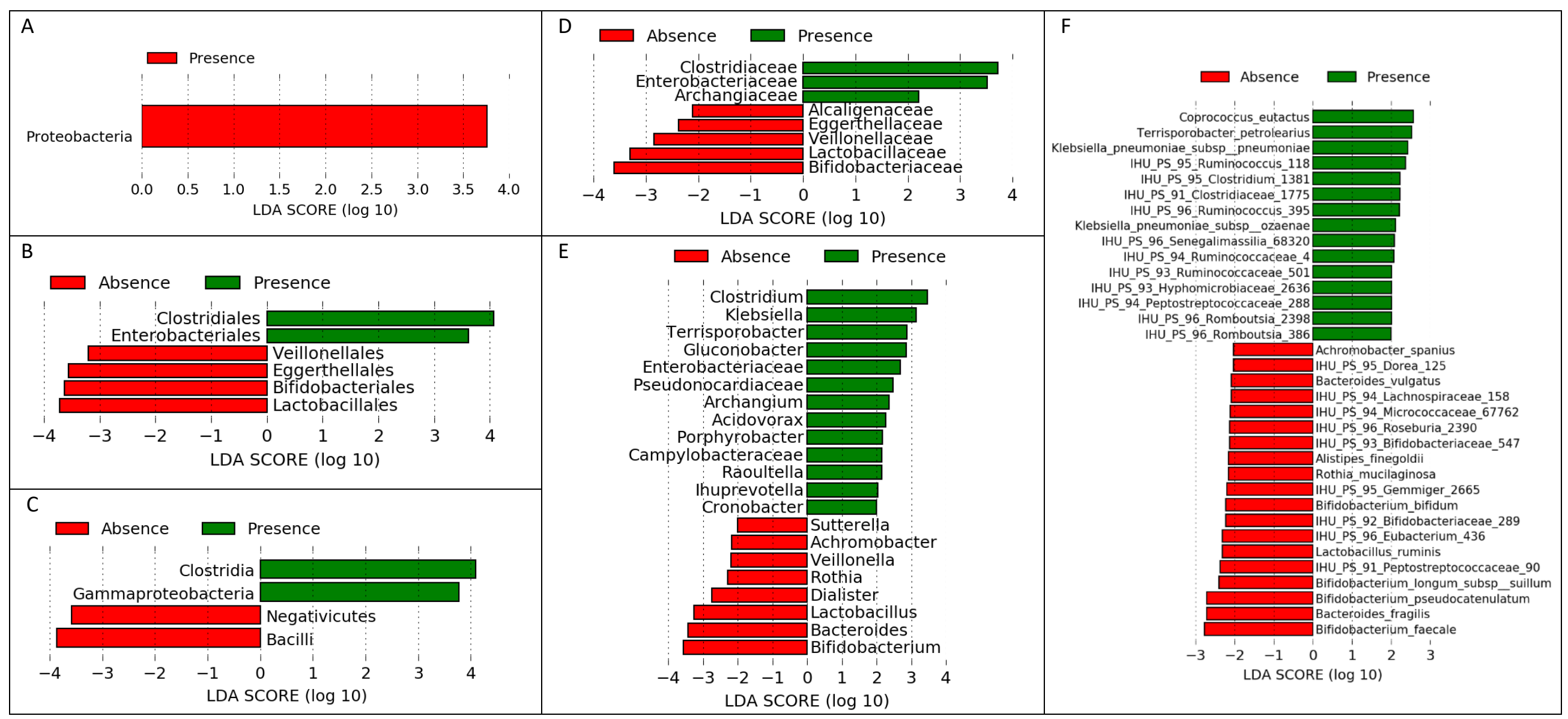

3 Figure 7. Linear size effect discriminant analysis (LDA LEfSe) between of bacterial gut microbiota of children who developed (green) or did not develop (red bars) at least one asymptomatic Plasmodium parasitaemia episode within 16 months of follow-up at the level of taxonomic classes 5 of the bacterial community structure at, A) Phyla, B) Order, C) Class, D) Family, E) genus, and F) species levels. Horizontal bars represent the 6 effect size for each taxon. The length of the bar represents the $\log 10$ transformed LDA score, indicated by vertical dotted lines. 
7

8

9

\subsection{Gut fungal community structure associated with malaria risk}

The alpha diversity indices did not statistically significantly differ between the children who developed at least one malaria attack (Table S4), asymptomatic Plasmodium parasitæmia episode (Table S5) and those who did not (Figure S11).

The beta diversity of the fungal community between children with at least one malaria attack $(n=105)$ and children tested negative $(n=191)$ was analysed by Principal Coordinated Analysis (PCoA) based on the Bray-Curtis similarity index. In line with a non-statistically significant ( $\mathrm{p}=0.27$ ) PERMANOVA test, PCoA showed no pattern (Figure 8a). Similar results were observed between the children who developed at least one asymptomatic Plasmodium parasitæmia episode $(n=81)$ and those $(n=215)$ who did not (PERMANOVA test, $p=0.76)$ (Figure 8b).

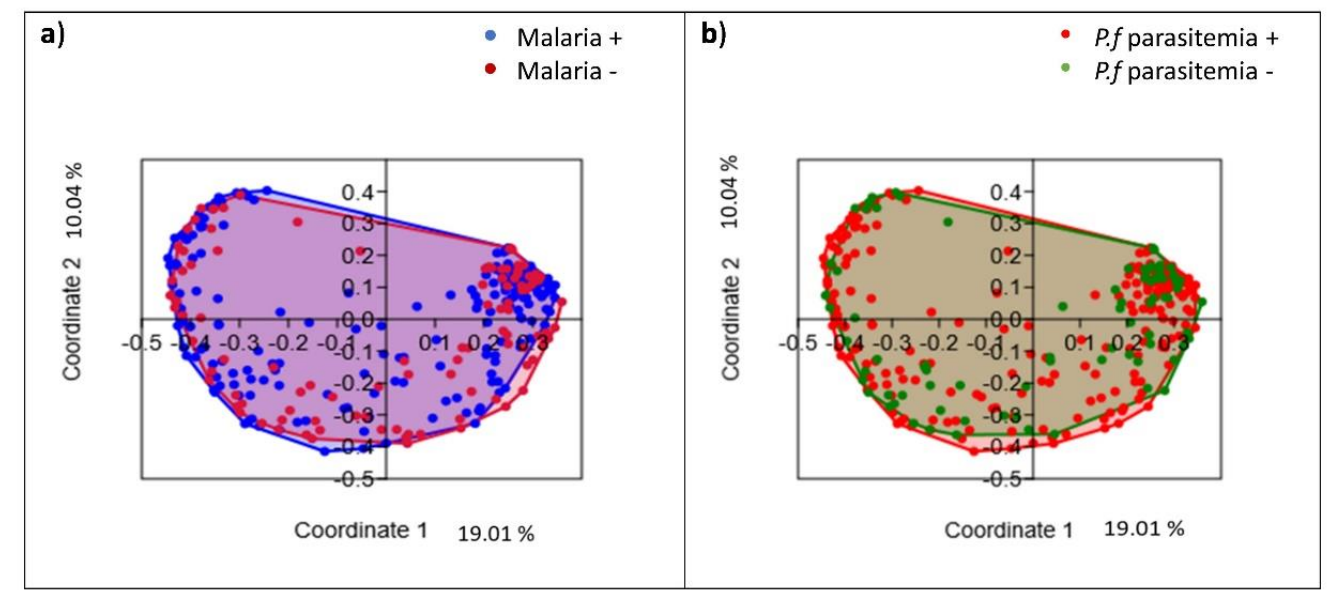

Figure 8. Gut fungal community and malaria risk. Principal Coordinates Analysis (PCoA) of the gut fungal community structure a) in children who experienced at least one malaria attack (crimson dots) or those who did not (blue dots) (PERMANOVA test, $\mathrm{p}=0.27$ ); $\mathbf{b}$ ) in children who developed at least one episode of Plasmodium parasitaemia (red dots) or did not (green dots) (PERMANOVA test, $\mathrm{p}=0.76$ ).

\subsection{Gut fungal community structure}

The relative mean of phyla abundance in children who developed at least one malaria attack was Ascomycota (46\%), Basidiomycota (62\%) compared to children with no malaria attack 
26 (Figure S12). In children who developed at least one asymptomatic Plasmodium parasitæmia episode, the relative mean of phyla abundance was Ascomycota (52\%), Basidiomycota (50\%) against children without an asymptomatic Plasmodium parasitæmia episode (Figure S13). At the phyla and class level, no abundance of fungi was significant between children who developed at least one attack of malaria and those who did not.

However, the most significantly abundant fungi in children who developed at least one malaria attack were the orders Glomerelalles, Families Turolaceae, genera Dioszegia, Turola, Cutaneotrichosporon and Geotrichum, species Dioszegia_fristingensis, Ogataea_polymorpha, Cutaneotrichosporon_cyanovorans, Unclassified_Geotrichum, Kluyveromyces_lactis, Torula_herbarum and Talaromyces_veerkampii (Figure 9A-D). The gut fungi of children who developed an asymptomatic episode of Plasmodium parasitæmia was comparable on the phyla level with those who did not. Regarding children who did not develop at least one attack of malaria, the Pezizaceae and Niessliaceae families, the Niesslia and Unclassified Aspergillaceae genera, the Didymocrea leucaenae, Niesslia exosporioides species and Unclassified Malassezia were the most significantly abundant (Figure 9B, 9C, 9D). 


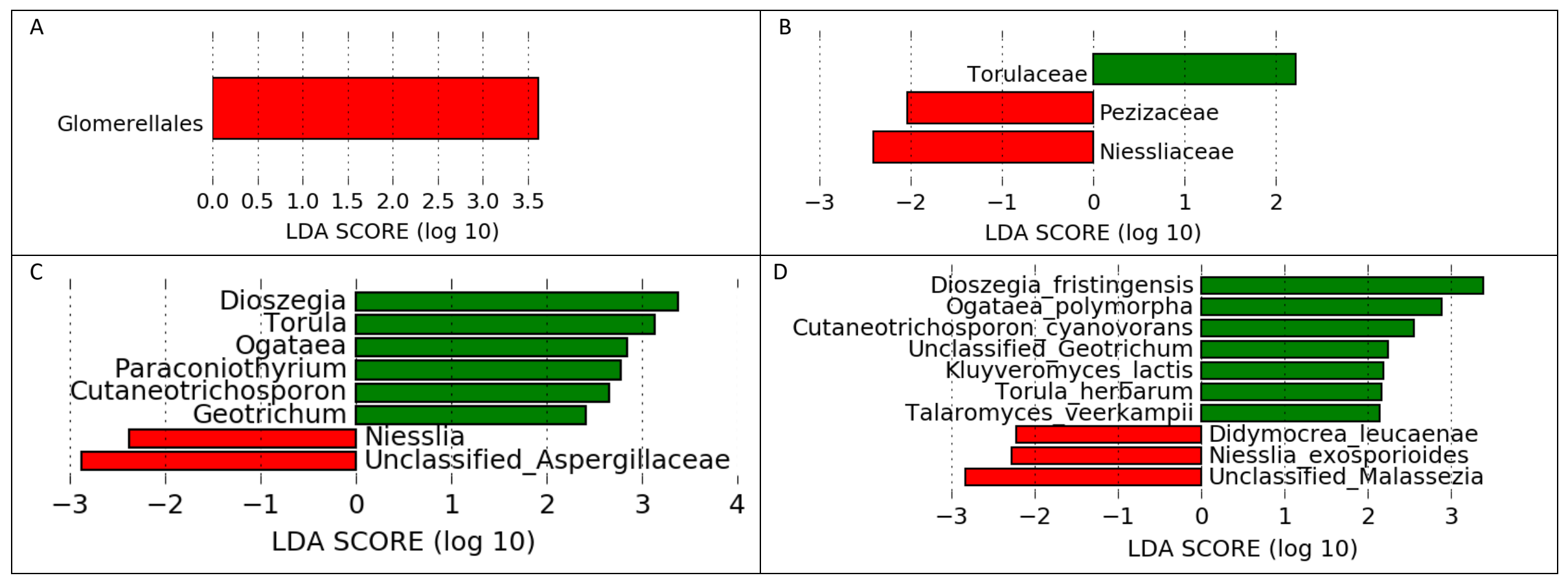

41 Figure 9. Linear size effect discriminant analysis (LDA LEfSe) of the fungal community structure at B) family, C) genus, and D) species levels, between children who experienced (green), or did not experience (red), at least one malaria attack within 16 months of follow-up. For A) Order children who experienced at least one malaria attack within 16 months of follow-up (red) were significant. Horizontal bars represent the effect size for each taxon. The length of the bar represents the log 10 transformed LDA score, indicated by vertical dotted lines. 
45 Considering the gut fungi of children who developed an asymptomatic episode of Plasmodium 46 parasitæmia, the classes Wallemiomycetes and Xylonomycetes, the Wallemial and 47 Symbiotaphrine Orders, the families Wallemiaceae, Tremellaceae, Symbiotaphrinaceae and 48 Lyophyllaceae, genera such as Wallemia, Exserohilum and Pseudoacremonium, and species 49 such as Wallemia mellicola, Unclassified Pseudoacremonium and Exserohilum antillanum 50 were the most abundant (Figure 10A-E). The gut fungal community of children who did not 51 develop an asymptomatic Plasmodium parasitæmia episode was characterised by the relative 52 abundance of the Cryptococcaceae families, and the presence of Leucosporidium yakuticum, 53 Aspergillus sydowii and Cryptococcus neoformans species (Figure 10C, 10E). 


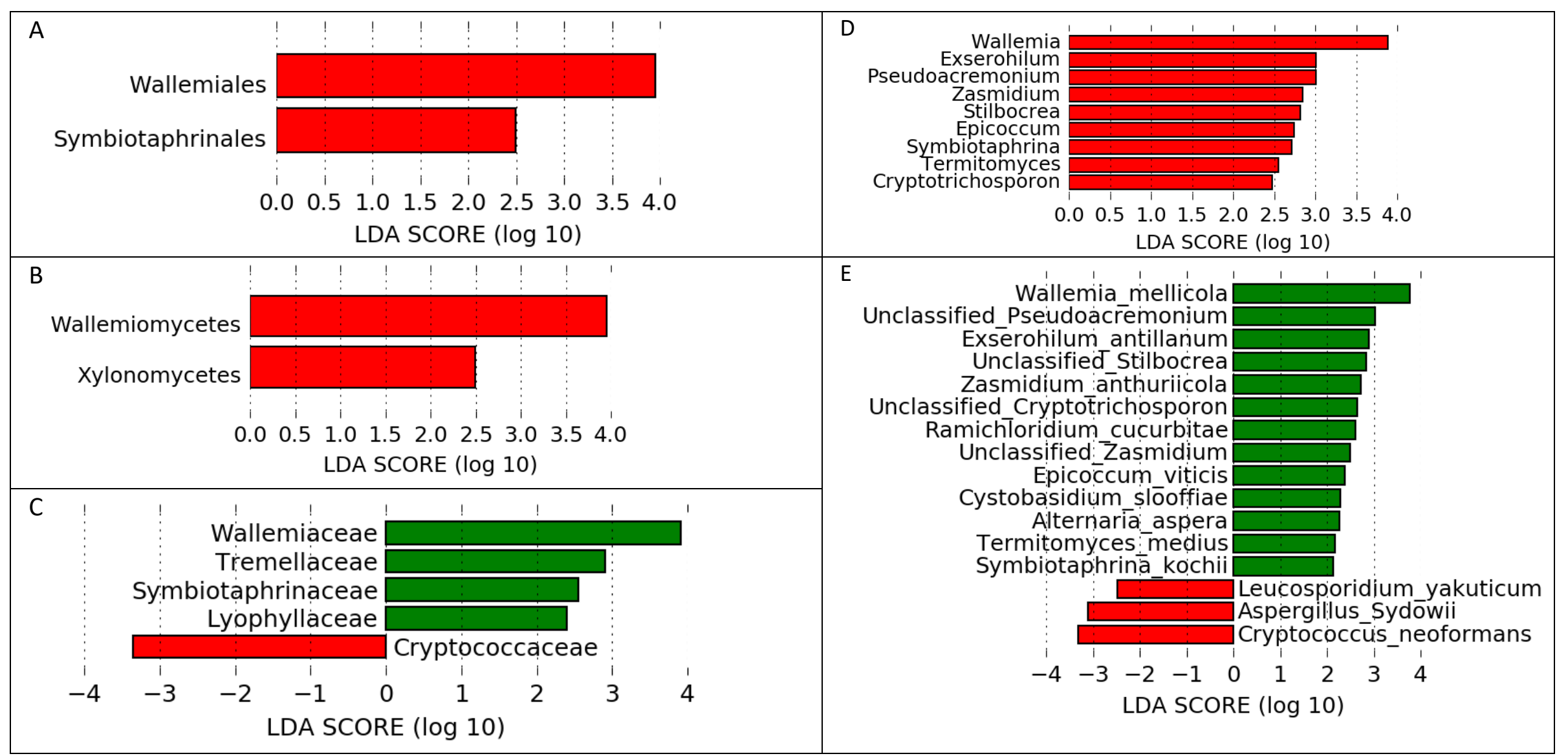

Figure 10. Linear size effect discriminant analysis (LDA LEfSe) of the fungi community structure at, C) Family and E) species levels, between children who developed (green), or did not develop (red), at least one asymptomatic Plasmodium parasitaemia episode within 16 months of followup. Fungi community structure at A) Order, B) Class and D) genus were significant in children who developed (red) at least one asymptomatic represents the $\log 10$ transformed LDA score, indicated by vertical dotted lines. 


\section{Discussion}

The findings of this study highlight the association of the gut bacteria but not fungi, community structures and specific bacteria and fungi taxa with susceptibility/resistance to malaria in children in an endemic area in Mali. The main limitations are a) the gut microbiome was analysed only at the baseline time point of the study, and it is possible that significant unmeasured variations occurred during the 16-month follow-up period; indeed, studies have shown that the gut microbiota varies according to season, lifestyle and diet [20,21]; b) the lack of information on the diet and nutritional status of children, which influence the gut microbiota, may expose the analysis to an unmeasured bias; c) the relatively small number of Fulani children included precludes any robust analysis of the differences compare to the Dogon ethnic group, that have been highlighted in previous studies on the Dogon [5,22]. Nevertheless, we found that Bacteroidetes were more abundant in Fulani children, while Actinobacteria were more abundant in Dogon children. Similarly, a relatively high abundance of Bacteroidetes has been observed in traditional societies practicing a hunter-gatherer lifestyle [23].

The main strengths of our study are a) the relatively large number of children included b) the precise identification of malaria attacks and, more particularly, of asymptomatic Plasmodium parasitæmia episodes through monthly qPCR testing during the 16 months of follow-up, and d) the analysis of both bacterial and fungal communities coupled with the qPCR detection of the major eukaryotic enteric pathogen to characterise the children's gut microbiomes. Regarding the epidemiology of malaria, our observation that children under five years old presented fewer malarial attacks than older children, was in line with a previous study conducted in the same locality, which found fewer malaria attacks in children under two years when compared to children aged three and four [24]. Furthermore, another study in a location in Senegal where seasonal malaria chemoprevention had not been implemented, showed that the prevalence of Plasmodium parasitæmia was $18 \%$ in children under five years and $25 \%$ in those aged between five and nine years [25]. It is notable that the children included in our study were not exposed to seasonal malaria chemoprevention, which has been associated with a significant reduction of malaria incidence in children under the age of five [26,27]. However, they did use both longlasting insecticidal nets (LLINs) and indoor residual spraying of insecticide (IRS), which might explain the observed higher malaria burden in older children [28]. Indeed, age was an independent factor that was strongly associated with both malaria attack and asymptomatic Plasmodium parasitæmia (Tables 2, S2). 
91 When investigating the gut microbiota associated susceptibility/resistance to malaria, we showed that the risks of both malaria attack and asymptomatic Plasmodium parasitæmia significantly increased with the increase in bacteria OTU richness (Tables 2, S2). These findings were confirmed by the survival analysis which found a higher risk of both malaria attack and asymptomatic Plasmodium parasitæmia in children with a bacteria OTU richness above the population's median value and in children over the age of five. A previous study in Mali found that the gut bacterial community structure was associated with asymptomatic Plasmodium parasitæmia but not with malaria attacks [19]. Another study in Kenya found no significant impact on the gut bacteria community structure before and after a malaria attack treated with arthemether/lumefantrine, although the authors detected sequence variants of some taxa that these episodes might have selected [29]. Several pieces of evidence point to a significant influence of the gut bacterial community on the risk of malaria infection. In a mouse model, severe Plasmodium infections have altered host gut homeostasis, which may contribute to malaria-related enteric bacteraemia [17]. Conversely, the gut bacterial microbiota composition can modulate the severity of $P$. yoelii $17 \mathrm{XNL}$ infection in mice and malaria susceptibility/resistance was transferred to germ-free mice by transferring the cecum contents of susceptible or resistant mice [2]. Our study further highlighted bacterial taxa could be differentiated between children who were susceptible or resistant to malaria attacks. Among these taxa, Clostridiacea, Lachnospiraceae, Intestinibacter, Actinomycetaceae, Slackia, Gordonibacter, Selenomonadaceae and Blastococcus were associated with susceptibility, while Bifidobacterium, Weissella, Veillonella, and Streptococcus timonensis were associated with resistance to malaria attacks. Furthermore, in children who were asymptomatic for malaria infection, the Proteobacteria phylum and Clostridium, Klebsiella, and Enterobacteriaceae were associated with susceptibility, while Bifidobacterium and Lactobacillus were associated with resistance to asymptomatic Plasmodium parasitæmia. Interestingly, known "beneficial" probiotic bacteria, such as Bifidobacterium and Lactobacillus were associated with malarial resistance [30,31].

To the best of our knowledge, our study is the first to investigate the association of the gut fungal community with the risk of malaria. We found that the gut fungal community structure was relatively homogenous between children who were susceptible to malaria and those who were resistant to it. This finding contrasts with previous reports of a higher abundance and diversity in the gut fungal community in patients with various diseases than in healthy subjects [32]. Likewise, gut fungal community dysbiosis has been associated with irritable bowel disease [33]. An unexpected finding was the association of the human opportunistic 
125 basidiomycete yeast, Cryptococcus neoformans, with resistance to Plasmodium parasitæmia.

126 Further studies are warranted to confirm the implication of the fungal taxa that have been found 127 to be associated with susceptibility/resistance to malaria in this exploratory study.

128

129

130

131

132

133

134

135

136

137

138

139

140

\section{Methods}

\subsection{Patient recruitment and specimen collection}

A longitudinal cohort study has been conducted from October 2017 to December 2018 in Bandiagara Malaria Project (BMP) clinical research centre in Mali. This period consisted of a season of low transmission from January to June and a season of seasonal and intense malaria transmission from July to December. The study involved children aged from six months to years who were not taking drugs with known antimalarial activity or antibiotics and who had no clinical symptoms of malaria. Recruited children have been monthly monitored for asymptomatic Plasmodium parasitæmia and, whenever a child presented with malaria symptoms, for a possible malaria attack. A clinical examination and a thick blood film and a blood drop on blotting paper were collected at each of each time point visit.

Children faeces were collected at Day-0 in identified sterile jars and immediately placed at $4{ }^{\circ} \mathrm{C}$. Hard stools were diluted v/v with 10X PBS (Phosphate-Buffered Saline pH 7.4, RNase-free) solution. Stool aliquots were distributed into $1 \mathrm{ml}$ tubes, kept at $-20^{\circ} \mathrm{C}$ in Mali, then packed in dry ice and shipped to Marseille for further 16S and ITS metabarcoding and qPCR detection of eukaryotic enteric pathogens.

\subsection{Malaria case definitions}

Malaria attack was defined by the detection of Plasmodium sp., at any density in at least one thick blood smear, and the presence of compatible clinical symptoms, including fever (body $\mathrm{T}^{\circ}>37.5^{\circ} \mathrm{C}$ ) or other malaria-associated symptoms. The thick smear was stained with $5 \%$ Giemsa and the number of parasites for 300 leukocytes was counted under a light microscope. Parasite density was estimated by calculating the number of asexual forms of Plasmodium $/ \mu 1$ of blood under the assumption that the leukocyte count was $7500 / \mu \mathrm{l}$. All children in whom a malaria attack was diagnosed were given antimalarial treatment according to the guidelines of the National Malaria Control Programme in Mali.

Asymptomatic Plasmodium parasitæmia was defined by the detection Plasmodium sp. DNA by qPCR on blood samples collected on blotting paper during the monthly monitoring of asymptomatic children. The identified blotting papers were dried, sealed in sachets with 
156 desiccants and stored at room temperature. Total DNA was extracted using the EZ1 DNA tissue 157 kit (Qiagen $\mathrm{GmbH}$, Hilden, Germany). Blotting paper fragments were inserted into a $1.5 \mathrm{ml}$ 158 tube containing $350 \mu 1$ of G2 lysis buffer and allowed to diffuse for 20-30 minutes. The tubes 159 containing the blotting paper were incubated at $100^{\circ} \mathrm{C}$ for 10 minutes and shaken briefly and 160 then centrifuged at $10,000 \mathrm{~g}$ for 10 minutes, before removing $200 \mu \mathrm{l}$ of the supernatant. A 161 mixture of the supernatant with $10 \mu 1$ of Proteinase $\mathrm{K}$ was incubated at $55^{\circ} \mathrm{C}$, for either two 162 hours or overnight. Total DNA was obtained using the EZ1 Advanced XL (QIAGEN 163 Instruments Hombrechtikon, Switzerland) with the DNA card bacteria V 1.066069118 164 QIAGEN and the EZ1 DNA tissue kit according to the manufacturer's procedures. The 165 extracted total DNA was stored at $4{ }^{\circ} \mathrm{C}$ and immediately used for the detection of Plasmodium 166 species. The extracted DNA was analysed by qPCR using the CFX96TM and CFX384TM 167 Real-Time PCR Detection Systems (BIO-RAD, Life Science, Marnes-la-Coquette, France) 168 using the following probes and primers (Table 3). The amplification reaction consisted of 10 $169 \mu \mathrm{L}$ Master Mix (Roche Diagnostics GmbH, Mannheim, Germany), $0.5 \mu \mathrm{L}$ of each primer, 0.5 $170 \mu \mathrm{L}$ of probe, $3 \mu \mathrm{L}$ of distilled water, $0.5 \mu \mathrm{L}$ of UDG and $5 \mu \mathrm{L}$ of DNA for a total volume of 20 $171 \mu \mathrm{L}$. The amplification programme followed consisted of two minutes at $50^{\circ} \mathrm{C}$ and five minutes 172 at $95^{\circ} \mathrm{C}$, followed by 40 cycles of five seconds at $95^{\circ} \mathrm{C}$ and one minute at $60^{\circ} \mathrm{C}$. The 173 amplification solution without a DNA template was the negative control; the positive control 174 was DNA from samples from patients in whom a Plasmodium sp. (P. falciparum, P. ovale, $P$. 175 malaria or $P$. vivax) has been documented. Samples with a cycle cut-off (Ct) less than 40 were 176 considered positive and confirmed by qPCR analysis. 
177 Table 3. Detail of the PCR primers and probes used in this study.

\begin{tabular}{|l|l|l|l|}
\hline Organisms & Gene & $\begin{array}{l}\text { Primers and } \\
\text { Probes }\end{array}$ & Sequences (5'-3') \\
\hline Plasmodium falciparum & $18 S$ & Pfalci_F & TAGCATATATTAAAATTGTTGCAG \\
\hline Plasmodium falciparum & & Pfalci_R & GTTATTCCATGCTGTAGTATTCA \\
\hline Plasmodium falciparum & & Probe & 6FAM- CGGGTAGTCATGATTGAGTTCATTC \\
\hline Plasmodium malariae & $18 S$ & Pmal_F & TAGCATATATTAAAATTGTTGCAG \\
\hline Plasmodium malariae & & Pmal_R & GTTATTCCATGCTGTAGTATTCA \\
\hline Plasmodium malariae & & Probe & 6FAM- TTGCATGGGAATTTTGTTACTTTGAGT \\
\hline Plasmodium ovale & $18 S$ & Pova_F & TAGCATATATTAAAATTGTTGCAG \\
\hline Plasmodium ovale & & Pova_R & GTTATTCCATGCTGTAGTATTCA \\
\hline Plasmodium ovale & & Probe & 6VIC- TGCATTCCTTATGCAAAATGTGTTC \\
\hline Plasmodium vivax & $18 S$ & Pviva_F & TAGCATATATTAAAATTGTTGCAG \\
\hline Plasmodium vivax & & Pviva_R & GTTATTCCATGCTGTAGTATTCA \\
\hline Plasmodium vivax & & Probe & 6VIC- CGACTTTGTGCGCATTTTGC \\
\hline Plasmodium sp. & Coxl & Plasmo_cox_15_F & AGGAACTCGACTGGCCTACA \\
\hline Plasmodium sp & & Plasmo_cox_16_R & CCAGCGACAGCGGTTATACT \\
\hline Plasmodium sp & & Probe & 6FAM- CGAACGCTTTTAACGCCTGACATGG \\
\hline
\end{tabular}

18S : 18S rRNA subunit coding gene

COX1: cytochrome $\mathrm{C}$ oxidase subunit 1 coding gene.

\subsection{Detection of intestinal parasites}

Aliquots of stools cryopreserved at $-80^{\circ} \mathrm{C}$ in the Marseille laboratory were subjected to total DNA extraction by the semi-automated method of EZ1 Advanced XL (QIAGEN Instruments Hombrechtikon, Switzerland) with the DNA card bacteria V 1.066069118 QIAGEN and the EZ1 DNA tissue kit following the procedure described by the manufacturer. Real-time PCR was performed on DNA extracted by the thermal cyclers of CFX96 ${ }^{\mathrm{TM}}$ and CFX $384^{\mathrm{TM}}$ RealTime PCR Detection Systems (BIO-RAD, Life Science, Marnes-la-Coquette, France) for the detection of 20 intestinal parasites following the procedure detailed below [34,35].

\subsection{Bacterial 16S metabarcoding analysis}

Stool samples were mechanical lysed using acid washed glass bead powder (G4649-500g Sigma) and $0.5 \mathrm{~mm}$ glass beads Cell rupture medium (Scientific Industries, Inc.) using a FastPrep BIO 101 instrument (Qbiogene, Strasbourg, France) at maximum speed $(6.5 \mathrm{~m} / \mathrm{sec})$ for 90 seconds. The DNA was then extracted following the procedures of two commercial kits, NucleoSpin Tissue (Macherey Nagel, Hœrdt, France) and method 5 using a deglycosylation step and purification on the EZ1 Advanced XL (Qiagen, Courtabœuf, France). The DNA extracted by these two procedures for each sample were then pooled and amplified PCR for 45 
196

197

198

199

200

201

202

203

204

205

206

207

208

209

210

211

212

213

214

215

216

217

218

219

220

221

222

223

224

225

226

227

cycles, with the Kapa HiFi Hotstart ReadyMix 2x reagents (Kapa Biosystems Inc., Wilmington, MA U.S.A.) and V3_V4 primers from the surrounding conserved region with adapters (FwOvAd_341F

\section{TCGTCGGCAGCGTCAGATGTGTATAAGAGACAGCCTACGGGNGGCWGCAG;} RevOvAd_785R

\section{GTCTCGTGGCTCGGAGATGTGTATAAGAGACAGGACTACHVGGGTATCTAATCC).}

The MiSeq system (Illumina, Inc., San Diego CA 92121, USA) with a paired end-of-sequence strategy allowed the sequencing of the 16S RNA gene of the V3-V4 hypervariable region according to the procedure used at IHU-MI platform [34]. The paired reads were filtered according to the read qualities. The raw data were configured in fastaq files for R1 and R2 reads. Analyses of the Reads were performed by the pandaseq tool and the vsearch tool. Clustering of the data was carried out by the Qiime tool. The SILVA and IHU-MI Culturomics $16 \mathrm{~S}$ databases were queried for taxonomic assignment of OTUs. The criteria established for taxonomic assignment of OTUs were as follows: 1) presence of one or more blast hits associated with a reference sequence $(100 \%$ coverage; identity $>97 \%$ corresponds to the assignment of OTUs to the species associated with the best blast hit); 2) presence of less relevant blast hits (identity between 95 and 97\%: assignment to genus level; between 90 and 95\%: assignment to the family; below 90\%: assignment to the kingdom) with, in each case, the creation of a putative species; 3 ) no blast hits (creation of putative new bacterial species). The analysis protocol was carried out by a bioinformatics company XEGEN [36].

\subsection{Fungal ITS1 and ITS2 metabarcoding}

The semi-automatic extraction protocol of EZ1 Advanced XL (QIAGEN Instruments Hombrechtikon, Switzerland) with the DNA card bacteria V 1.066069118 QIAGEN and the EZ1 DNA tissue kit was used to extract the total DNA as detailed here[34]. The amplification reaction mix consisted of $12.5 \mu \mathrm{l}$ AmpliTaq Gold master mix, $0.75 \mu \mathrm{l}$ of each primer (Eurogentec, Seraing, Belgium), $6 \mu 1$ distilled water and $5 \mu 1$ DNA template for $25 \mu 1$ volume. The amplification programme was as follows: $95^{\circ} \mathrm{C}$ for 10 minutes, $95^{\circ} \mathrm{C}$ for 30 seconds, $\left(55^{\circ} \mathrm{C}\right.$ or $52^{\circ} \mathrm{C}$ ) for 30 seconds, $72^{\circ} \mathrm{C}$ for one minute, and $72^{\circ} \mathrm{C}$ for five minutes. Amplification of the ITS1 and ITS2 region using the primers described herein [32] with independent hybridisation temperatures of $52^{\circ} \mathrm{C}$ and $55^{\circ} \mathrm{C}$, were made in triplicate. The amplicons of the replicated PCRs and the two hybridisation temperatures relating to ITS1 and ITS2 were pooled for metabarcoding on the MiSeq platform. 
After purification on AMPure beads (Beckman Coulter Inc., Fullerton, CA, USA), concentration was measured using high sensitivity Qubit technology (Beckman Coulter Inc., Fullerton, CA, USA) and dilution to $3.5 \mathrm{ng} / \mu 1$ was performed. At this step, Illumina sequencing adapters and dual-index barcodes were added to the amplicon. After purification on AMPure beads (Beckman Coulter Inc., Fullerton, CA, USA), this library was pooled with 94 other multiplexed samples. The global concentration was quantified by a Qubit assay with the high sensitivity kit (Life technologies, Carlsbad, CA, USA). Before loading for sequencing on MiSeq (Illumina Inc., San Diego, CA, USA) the pool was diluted at 8pM. Automated cluster generation and paired-end sequencing with dual index reads was performed in a single 39-hour run in a $2 \times 250 \mathrm{bp}$. The paired reads were filtered according to the read qualities. The raw data were configured in fastaq files for $\mathrm{R} 1$ and $\mathrm{R} 2$ reads.

The Illumina MiSeq sequences analysis was performed by PIPITS, an automated pipeline for the analysis of fungal ITS (internal transcribed spacer) sequences from the Illumina sequencing platform, hereafter referred to as the protocol [37]. The pipeline consists in the following consecutive steps: 1) preparation of raw sequences (joining, conversion, quality filtering, relabelling and file formatting), 2) extraction of ITS fungi and read re-orientation, 3) processing of the reads to produce an operational taxonomy unit (OTU) abundance table and taxonomic assignment table for downstream analysis. In this case, the extracted ITS2 and ITS1 sequences were analysed for the processes to obtain the OTU table. The OTU sequences were defined as a cluster of $97 \%$ sequence identity. The last step generated the repseqs.fasta file representing the OTU sequences. These OTU sequences were manually queried via BLASTN against the nucleotide NCBI with the search parameters: 1) rRNA genes internal transcribed spacer region (ITS) from fungi type and reference material and, if the first query yielded <97\% identity, 2) the nucleotide collection (nt) to improve the taxonomic assignment that was generated by PIPITS. The taxon selection criteria were defined as follows: PID (percentage of identity) > 97\% assignment to species; PID between 95 and 97\%: assignment to genus level; PID between 90 and 95\%: assignment to the family; PID below 90\%: assignment to the kingdom.

\subsection{Statistical analysis}

The covariates were described via median, interquartile range, mean, and standard deviation computed with the GraphPad Prism ver. 5.03 for Windows software. Two malaria phenotypes were analysed: 1) the children who developed at least one malaria attack were compared to those who developed no malaria attack, and 2) the children who developed at least one 
260

261

262

263

264

265

266

267

268

269

270

271

272

273

274

275

276

277

278

279

280

281

282

283

284

285

286

287

288

289

290

asymptomatic Plasmodium parasitæmia episode, were compared to those who developed no Plasmodium parasitæmia episode during the 16 months of follow-up. Because several studies have indicated that Fulani people were less susceptible to malaria than Dogon people [4,5] we tested whether malaria susceptibility/resistance differed between the children of these two sympatric ethnic groups. A logistic regression model of SPSS 12.0 for Windows was used to assess the relationship between gut bacteria and fungi community structure and malaria infection by adjusting for age, gender, ethnicity, and the presence of eukaryotic enteric pathogens. In this analysis, the children with asymptomatic Plasmodium parasitæmia at baseline, were excluded. The following transformations: square-root, square, and natural logarithm of the continuous variables, including age and alpha diversity indices of the bacterial and fungal communities, were tested and the best-fitting transformation (assessed via Akaike Information Criterion) of each was used in the logistical regression model. The log-rank (Mantel-Cox) test was used to compare the survival distributions between groups with GraphPad Prism. The PAST4 software (PAleontological STatistics Version 4.01) was used to compare groups in terms of diversity (Shannon and Simpson indices) and richness (Chao-1 indices, number of observed OTUs) of bacterial and fungal species, and to explore the beta diversity between children with distinct malaria phenotypes via a Principal coordinate analysis (PCoA) graph and the non-parametric PERMANOVA (Permutational Multivariate Analysis of Variance). The comparison of the microbiota community between groups was carried out using the Linear Size Effect Discriminant Analysis (LDA LEfSe) available at http://huttenhower.sph.harvard.edu/galaxy/. All statistical tests were two-tailed; statistical significance threshold was set at $\mathrm{P}<0.05$.

\subsection{Ethical declaration}

This study was approved by the Ethics Committee of the Faculty of Medicine of Mali (N²017/133/CE/FMPOS). Each child included, and/or at least one of their parents or guardians gave their informed, written consent to participate in the study.

\section{Conclusions}

The results of this study showed that the gut bacterial community structure, but not the fungal community, is associated with susceptibility/resistance to malaria attacks and asymptomatic $P$. falciparum malaria infection. We demonstrated that gut bacteria OTU richness was independently associated with the risk of a malaria attack. This points the way towards 
291 strategies aiming to reduce malaria risk in endemic areas by modulating the gut microbiota components of at-risk populations.

293

294

295

296

297

298

299

300

301

302

303

304

305

306

307

308

309

310

311

312

313

314

315

316

317

318

319

320

321

322

\section{Author's contributions}

O.K. DOUMBO, D. RAOULT, S. RANQUE and A. KODIO designed and conceived the study. D. COULIBALY, A.K. KONÉ, S. KONATÉ, B. GUINDO, S. DOUMBO, and M.A. THERA included the study participants, and performed clinical and biological evaluations, sample collection and data management. A. KODIO, A.M. KONATÉ and C. L'OLLIVIER performed the laboratory work. A. KODIO, L. TALL, A. LEVASSEUR, S. RANQUE, and F. BITTAR did the bioinformatics and statistical analysis. The manuscript was drafted by A. KODIO and edited by S. RANQUE. All authors read and approved the final version of the manuscript.

\section{Acknowledgments}

The authors are grateful to the authorities and the people of Bandiagara for their participation in the study. They acknowledge the technical assistance of Caroline Blanc-Tailleur and Enora Tomeï for the 16S, ITS1, ITS2 Illumina MiSeq metagenomics analysis. Also, they are grateful to Sylvain Buffet for his computer assistance.

\section{Funding details}

This work was supported by the French Government under the 'Investissements d'avenir' (Investments for the Future) programme managed by the Agence Nationale de la Recherche (ANR, fr: National Agency for Research), (reference: Méditerranée Infection 10-IAHU-03), the IHU-Mediterranean Infection Foundation, and the MARCAD DELTAS Africa Initiative grant DEL-15-10. The DELTAS Africa Initiative is an independent funding scheme of the African Academy of Sciences' (AAS) Alliance for Accelerating Excellence in Science in Africa (AESA) and supported by the New Partnership for Africa's Development Planning and Coordinating Agency (NEPAD Agency) with funding from the Wellcome Trust grant 107741/A/15/Z and the UK government. The views expressed in this publication are those of the author(s) and not necessarily those of the AAS, the NEPAD Agency, the Wellcome Trust, or the UK government.

\section{Disclosure Statement}

The authors report no conflict of interest.

\section{Data availability}

The data generated and analysed in this study are available on the website of the Institut Hospitalo-Universitaire - Méditerranée Infection (IHU- Méditerranée Infection). 
https://www.mediterranee-infection.com/acces-ressources/donnees-pour-articles/gutmicrobiota-influences-plasmodium-falciparum-malaria-susceptibility/

\section{References}

1. World Malaria Report 2019 Available online: https://www.who.int/publicationsdetail/world-malaria-report-2019 (accessed on 14 March 2020).

2. Villarino, N.F.; LeCleir, G.R.; Denny, J.E.; Dearth, S.P.; Harding, C.L.; Sloan, S.S.; Gribble, J.L.; Campagna, S.R.; Wilhelm, S.W.; Schmidt, N.W. Composition of the Gut Microbiota Modulates the Severity of Malaria. PNAS 2016, 113, 2235-2240, doi:10.1073/pnas.1504887113.

3. Milner, D.A. Malaria Pathogenesis. Cold Spring Harb Perspect Med 2018, 8, doi:10.1101/cshperspect.a025569.

4. Modiano, D.; Petrarca, V.; Sirima, B.S.; Bosman, A.; Nebié, I.; Diallo, D.; Lamizana, L.; Esposito, F.; Coluzzi, M. Plasmodium Falciparum Malaria in Sympatric Ethnic Groups of Burkina Faso, West Africa. Parassitologia 1995, 37, 255-259.

5. Dolo, A.; Modiano, D.; Maiga, B.; Daou, M.; Dolo, G.; Guindo, H.; Ba, M.; Maiga, H.; Coulibaly, D.; Perlman, H.; et al. Difference in Susceptibility to Malaria between Two Sympatric Ethnic Groups in Mali. Am. J. Trop. Med. Hyg. 2005, 72, 243-248.

6. Taylor, S.M.; Parobek, C.M.; Fairhurst, R.M. Haemoglobinopathies and the Clinical Epidemiology of Malaria: A Systematic Review and Meta-Analysis. Lancet Infect Dis 2012, 12, 457-468, doi:10.1016/S1473-3099(12)70055-5.

7. Travassos, M.A.; Coulibaly, D.; Laurens, M.B.; Dembélé, A.; Tolo, Y.; Koné, A.K.; Traoré, K.; Niangaly, A.; Guindo, A.; Wu, Y.; et al. Hemoglobin C Trait Provides Protection From Clinical Falciparum Malaria in Malian Children. J. Infect. Dis. 2015, 212, 1778-1786, doi:10.1093/infdis/jiv308.

8. Gunalan, K.; Niangaly, A.; Thera, M.A.; Doumbo, O.K.; Miller, L.H. Plasmodium Vivax Infections of Duffy-Negative Erythrocytes: Historically Undetected or a Recent Adaptation? Trends Parasitol. 2018, 34, 420-429, doi:10.1016/j.pt.2018.02.006.

9. Marsh, K.; Snow, R.W. Host-Parasite Interaction and Morbidity in Malaria Endemic Areas. Philos. Trans. R. Soc. Lond., B, Biol. Sci. 1997, 352, 1385-1394, doi:10.1098/rstb.1997.0124.

10. Tran, T.M.; Li, S.; Doumbo, S.; Doumtabe, D.; Huang, C.-Y.; Dia, S.; Bathily, A.; Sangala, J.; Kone, Y.; Traore, A.; et al. An Intensive Longitudinal Cohort Study of Malian Children and Adults Reveals No Evidence of Acquired Immunity to Plasmodium Falciparum Infection. Clin Infect Dis 2013, 57, 40-47, doi:10.1093/cid/cit174.

11. Gonçalves, B.P.; Huang, C.-Y.; Morrison, R.; Holte, S.; Kabyemela, E.; Prevots, D.R.; Fried, M.; Duffy, P.E. Parasite Burden and Severity of Malaria in Tanzanian Children. N. Engl. J. Med. 2014, 370, 1799-1808, doi:10.1056/NEJMoa1303944.

12. Crompton, P.D.; Moebius, J.; Portugal, S.; Waisberg, M.; Hart, G.; Garver, L.S.; Miller, L.H.; Barillas, C.; Pierce, S.K. Malaria Immunity in Man and Mosquito: Insights into Unsolved Mysteries of a Deadly Infectious Disease. Annu Rev Immunol 2014, 32, 157187, doi:10.1146/annurev-immunol-032713-120220.

13. Lyke, K.E. Steady Progress toward a Malaria Vaccine. Curr. Opin. Infect. Dis. 2017, 30, 463-470, doi:10.1097/QCO.0000000000000393.

14. Frimpong, A.; Kusi, K.A.; Ofori, M.F.; Ndifon, W. Novel Strategies for Malaria Vaccine Design. Front Immunol 2018, 9, 2769, doi:10.3389/fimmu.2018.02769.

15. Yilmaz, B.; Portugal, S.; Tran, T.M.; Gozzelino, R.; Ramos, S.; Gomes, J.; Regalado, A.; Cowan, P.J.; d'Apice, A.J.F.; Chong, A.S.; et al. Gut Microbiota Elicits a Protective Immune Response against Malaria Transmission. Cell 2014, 159, 1277-1289, doi:10.1016/j.cell.2014.10.053. 
16. Aguilar, R.; Ubillos, I.; Vidal, M.; Balanza, N.; Crespo, N.; Jiménez, A.; Nhabomba, A.; Jairoce, C.; Dosoo, D.; Gyan, B.; et al. Antibody Responses to $\alpha$-Gal in African Children Vary with Age and Site and Are Associated with Malaria Protection. Sci Rep 2018, 8, 9999, doi:10.1038/s41598-018-28325-w.

17. Denny, J.E.; Powers, J.B.; Castro, H.F.; Zhang, J.; Joshi-Barve, S.; Campagna, S.R.; Schmidt, N.W. Differential Sensitivity to Plasmodium Yoelii Infection in C57BL/6 Mice Impacts Gut-Liver Axis Homeostasis. Sci Rep 2019, 9, 3472, doi:10.1038/s41598-01940266-6.

18. Morffy Smith, C.D.; Gong, M.; Andrew, A.K.; Russ, B.N.; Ge, Y.; Zadeh, M.; Cooper, C.A.; Mohamadzadeh, M.; Moore, J.M. Composition of the Gut Microbiota Transcends Genetic Determinants of Malaria Infection Severity and Influences Pregnancy Outcome. EBioMedicine 2019, 44, 639-655, doi:10.1016/j.ebiom.2019.05.052.

19. Yooseph, S.; Kirkness, E.F.; Tran, T.M.; Harkins, D.M.; Jones, M.B.; Torralba, M.G.; O’Connell, E.; Nutman, T.B.; Doumbo, S.; Doumbo, O.K.; et al. Stool Microbiota Composition Is Associated with the Prospective Risk of Plasmodium Falciparum Infection. BMC Genomics 2015, 16, 631, doi:10.1186/s12864-015-1819-3.

20. Smits, S.A.; Leach, J.; Sonnenburg, E.D.; Gonzalez, C.G.; Lichtman, J.S.; Reid, G.; Knight, R.; Manjurano, A.; Changalucha, J.; Elias, J.E.; et al. Seasonal Cycling in the Gut Microbiome of the Hadza Hunter-Gatherers of Tanzania. Science 2017, 357, 802806, doi:10.1126/science.aan4834.

21. Wu, G.D.; Chen, J.; Hoffmann, C.; Bittinger, K.; Chen, Y.-Y.; Keilbaugh, S.A.; Bewtra, M.; Knights, D.; Walters, W.A.; Knight, R.; et al. Linking Long-Term Dietary Patterns with Gut Microbial Enterotypes. Science 2011, 334, 105-108, doi:10.1126/science.1208344.

22. C, A.; B, M.; A, D.; B, K.; B, T.; Pd, C.; Sk, P.; M, T.-B.; Lh, M.; Ok, D. Ethnic Differences in Susceptibility to Malaria: What Have We Learned from ImmunoEpidemiological Studies in West Africa? Acta Trop 2015, 146, 152-156, doi:10.1016/j.actatropica.2015.03.023.

23. Afolayan, A.O.; Ayeni, F.A.; Moissl-Eichinger, C.; Gorkiewicz, G.; Halwachs, B.; Högenauer, C. Impact of a Nomadic Pastoral Lifestyle on the Gut Microbiome in the Fulani Living in Nigeria. Front Microbiol 2019, 10, doi:10.3389/fmicb.2019.02138.

24. Coulibaly, D.; Travassos, M.A.; Kone, A.K.; Tolo, Y.; Laurens, M.B.; Traore, K.; Diarra, I.; Niangaly, A.; Daou, M.; Dembele, A.; et al. Stable Malaria Incidence despite Scaling up Control Strategies in a Malaria Vaccine-Testing Site in Mali. Malar J 2014, 13, doi:10.1186/1475-2875-13-374.

25. Ndiaye, J.L.A.; Ndiaye, Y.; Ba, M.S.; Faye, B.; Ndiaye, M.; Seck, A.; Tine, R.; Thior, P.M.; Atwal, S.; Beshir, K.; et al. Seasonal Malaria Chemoprevention Combined with Community Case Management of Malaria in Children under 10 Years of Age, over 5 Months, in South-East Senegal: A Cluster-Randomised Trial. PLoS Med. 2019, 16, e1002762, doi:10.1371/journal.pmed.1002762.

26. Druetz, T. Evaluation of Direct and Indirect Effects of Seasonal Malaria Chemoprevention in Mali. Scientific Reports 2018, 8, 8104, doi:10.1038/s41598-01826474-6.

27. Konaté, D.; Diawara, S.I.; Touré, M.; Diakité, S.A.S.; Guindo, A.; Traoré, K.; Diarra, A.; Keita, B.; Thiam, S.; Keita, M.; et al. Effect of Routine Seasonal Malaria Chemoprevention on Malaria Trends in Children under 5 Years in Dangassa, Mali. Malar. J. 2020, 19, 137, doi:10.1186/s12936-020-03202-y.

28. Kigozi, S.P.; Kigozi, R.N.; Epstein, A.; Mpimbaza, A.; Sserwanga, A.; Yeka, A.; Nankabirwa, J.I.; Halliday, K.; Pullan, R.L.; Rutazaana, D.; et al. Rapid Shifts in the 
Age-Specific Burden of Malaria Following Successful Control Interventions in Four Regions of Uganda. Malar. J. 2020, 19, 128, doi:10.1186/s12936-020-03196-7.

29. Mandal, R.K.; Crane, R.J.; Berkley, J.A.; Gumbi, W.; Wambua, J.; Ngoi, J.M.; Ndungu, F.M.; Schmidt, N.W. Longitudinal Analysis of Infant Stool Bacteria Communities Before and After Acute Febrile Malaria and Artemether-Lumefantrine Treatment. $J$ Infect Dis 2019, 220, 687-698, doi:10.1093/infdis/jiy740.

30. Turroni, F.; Milani, C.; Duranti, S.; Ferrario, C.; Lugli, G.A.; Mancabelli, L.; van Sinderen, D.; Ventura, M. Bifidobacteria and the Infant Gut: An Example of CoEvolution and Natural Selection. Cell. Mol. Life Sci. 2018, 75, 103-118, doi:10.1007/s00018-017-2672-0.

31. Huda, M.N.; Ahmad, S.M.; Alam, M.J.; Khanam, A.; Kalanetra, K.M.; Taft, D.H.; Raqib, R.; Underwood, M.A.; Mills, D.A.; Stephensen, C.B. Bifidobacterium Abundance in Early Infancy and Vaccine Response at 2 Years of Age. Pediatrics 2019, 143, doi:10.1542/peds.2018-1489.

32. Hamad, I.; Ranque, S.; Azhar, E.I.; Yasir, M.; Jiman-Fatani, A.A.; Tissot-Dupont, H.; Raoult, D.; Bittar, F. Culturomics and Amplicon-Based Metagenomic Approaches for the Study of Fungal Population in Human Gut Microbiota. Sci Rep 2017, 7, 16788, doi:10.1038/s41598-017-17132-4.

33. Sokol, H.; Leducq, V.; Aschard, H.; Pham, H.-P.; Jegou, S.; Landman, C.; Cohen, D.; Liguori, G.; Bourrier, A.; Nion-Larmurier, I.; et al. Fungal Microbiota Dysbiosis in IBD. Gut 2017, 66, 1039-1048, doi:10.1136/gutjnl-2015-310746.

34. Kodio, A.; Coulibaly, D.; Koné, A.K.; Konaté, S.; Doumbo, S.; Guindo, A.; Bittar, F.; Gouriet, F.; Raoult, D.; Thera, M.A.; et al. Blastocystis Colonization Is Associated with Increased Diversity and Altered Gut Bacterial Communities in Healthy Malian Children. Microorganisms 2019, 7, doi:10.3390/microorganisms7120649.

35. Sow, D.; Parola, P.; Sylla, K.; Ndiaye, M.; Delaunay, P.; Halfon, P.; Camiade, S.; Dieng, T.; Tine, R.C.K.; Faye, B.; et al. Performance of Real-Time Polymerase Chain Reaction Assays for the Detection of 20 Gastrointestinal Parasites in Clinical Samples from Senegal. Am J Trop Med Hyg 2017, 97, 173-182, doi:10.4269/ajtmh.16-0781.

36. XEGEN - The specialist in high performance and high throughput NGS data analysis and functional annotation.

37. Gweon, H.S.; Oliver, A.; Taylor, J.; Booth, T.; Gibbs, M.; Read, D.S.; Griffiths, R.I.; Schonrogge, K. PIPITS: An Automated Pipeline for Analyses of Fungal Internal Transcribed Spacer Sequences from the Illumina Sequencing Platform. Methods Ecol Evol 2015, 6, 973-980, doi:10.1111/2041-210X.12399. 


\section{Legends of tables and figures}

Table 1. Baseline characteristics of the children included in the cohort study, by age group.

Table 2. Logistic regression analysis of the association between age and gut bacterial and fungal community structure with the risk of malaria attack.

Table 3. PCR primer and probe sequences used in this study.

Figure 1. Relative frequency (A) and abundance (B) of the major gut bacterial phyla, and relative frequency (C) and abundance (D) of the major gut fungal phyla, characterised via 16S or ITS metabarcoding.

Figure 2. Distribution of the relative frequency A), and abundance B) of gut fungal phyla metabarcoding reads according to age. (Figures inside the bars are the mean abundance and number of the phylum for each group).

Figure 3. Gut bacterial community structure and malaria risk. Principal Coordinates Analysis (PCoA) of the gut bacterial community a) in children who experienced (yellow dots), or did not experience (blue dots), at least one malaria attack within 16 months of follow-up (Permanova test, $\mathrm{p}=0.0054$ ); $\mathrm{b}$ ) in children who developed (green dots), or did not develop (pink dots), at least one asymptomatic Plasmodium parasitaemia episode within 16 months of follow-up (Permanova test, $\mathrm{p}=0.012$ ).

Figure 4. Richness and diversity indices of the gut bacterial community in children who experienced or did not experience at least one malaria attack within 16 months of follow-up. Scatter dot plot and Box-Whisker's graph shows Median, Min and Max of children who experienced at least one malaria attack (green) compared to control (blue) A) for chao-1 index ( $\mathrm{p}=0.0356) ; \mathrm{B})$ for Shannon index $(\mathrm{p}=0.1411)$; C) for OTU Richness ( $\mathrm{p}=0.0012)$; D) for Simpson index $(\mathrm{p}=0.7996)$.

Figure 5. Richness and diversity indices of gut bacterial community of children who developed or did not develop at least one asymptomatic Plasmodium parasitaemia episode within 16 months of follow-up. Median and range of chao-1 index (p=0.049) a); of Shannon index $(\mathrm{p}=0.957) \mathrm{b})$; for OTU Richness $(\mathrm{p}=0.02) \mathrm{c})$ and Simpson index $(\mathrm{p}=0.395) \mathrm{d})$ of children who developed at least one asymptomatic Plasmodium parasitaemia episode (light blue) compared to control (pink) for Scatter dot plot, Box and Whiskers graph. 
Figure 6. Linear size effect discriminant analysis (LDA LEfSe) of the gut bacterial community structure at, a) Order, b) Family, c) Genus and d) species levels, between children who experienced (green), or did not experience (red), at least one malaria attack within 16 months of follow-up. Horizontal bars represent the effect size for each taxon. The length of the bar represents the $\log 10$ transformed LDA score, indicated by vertical dotted lines.

Figure 7. Linear size effect discriminant analysis (LDA LEfSe) between of bacterial gut microbiota of children who developed (green) or did not develop (red bars) at least one asymptomatic Plasmodium parasitaemia episode within 16 months of follow-up at the level of taxonomic classes of the bacterial community structure at, A) Phyla, B) Order, C) Class, D) Family, E) genus, and F) species levels. Horizontal bars represent the effect size for each taxon. The length of the bar represents the $\log 10$ transformed LDA score, indicated by vertical dotted lines.

Figure 8. Gut fungal community and malaria risk. Principal Coordinates Analysis (PCoA) of the gut fungal community structure a) in children who experienced at least one malaria attack (crimson dots) or those who did not (blue dots) (PERMANOVA test, $\mathrm{p}=0.27$ ); $\mathbf{b}$ ) in children who developed at least one episode of Plasmodium parasitaemia (red dots) or did not (green dots) (PERMANOVA test, $\mathrm{p}=0.76$ ).

Figure 9. Linear size effect discriminant analysis (LDA LEfSe) of the fungal community structure at B) family, C) genus, and D) species levels, between children who experienced (green), or did not experience (red), at least one malaria attack within 16 months of follow-up. For A) Order children who experienced at least one malaria attack within 16 months of follow-up (red) were significant. Horizontal bars represent the effect size for each taxon. The length of the bar represents the $\log 10$ transformed LDA score, indicated by vertical dotted lines.

Figure 10. Linear size effect discriminant analysis (LDA LEfSe) of the fungi community structure at, C) Family and E) species levels, between children who developed (green), or did not develop (red), at least one asymptomatic Plasmodium parasitaemia episode within 16 months of follow-up. Fungi community structure at A) Order, B) Class and D) genus were significant in children who developed (red) at least one asymptomatic Plasmodium parasitaemia episode within 16 months of follow-up. Horizontal bars represent the effect size for each taxon. The length of the bar represents the $\log 10$ transformed LDA score, indicated by vertical dotted lines. 


\section{Supplementary data}

Figure S1. Log-rank (mantel-Cox) test comparing the risk of experiencing at least one malaria attack (A) or at least one asymptomatic Plasmodium parasitemia episode (B) in children $\leq 4$ years-old $(n=74)$ or $>$ four years-old $(n=184)$, within 16 months of follow-up. The 35 children who presented with asymptomatic Plasmodium parasitaemia at baseline were excluded from this analysis.

Figure S2. Log-rank (mantel-Cox) test comparing the risk of experiencing at least one malaria attack (A) or at least one asymptomatic Plasmodium parasitaemia episode (B) in children with either low $(n=130)$ or high $(n=128)$ gut bacteria OTU richness, within 16 months of follow-up. The 35 children who presented with asymptomatic Plasmodium parasitaemia at baseline were excluded from this analysis. A) Fraction of malaria attack free; B) Fraction of no asymptomatic Plasmodium parasitemia. Children with low (red); high gut bacteria OTU richness (green).

Figure S3. Risk of developing within 16 months of follow-up: a) at least one malarial attack or b) at least one episode of asymptomatic Plasmodium parasitemia in 0 to 4 years old children with gut bacteria OTU richness below ( $n=38$, red line) or above ( $n=36$, green line) the median value.

Figure S4. Risk of developing within 16 months of follow-up: a) at least one malarial attack or b) at least one episode of asymptomatic Plasmodium parasitemia in 5 to 15 years old children with gut bacteria OTU richness below ( $\mathrm{n}=93$, red line) or above ( $\mathrm{n}=91$, green line) the median value.

Figure S5. Gut bacterial community structure. Relative abundance of phyla in children who experienced (yellow bars) or who did not experience (blue bars) at least one malaria attack within 16 months of follow-up. (Figures inside the bars are the mean abundance of the phylum for each group).

Figure S6. Gut bacterial community structure. Relative abundance of phyla in children who developed (yellow bars) or did not develop (blue bars) at least one asymptomatic Plasmodium parasitaemia episode within 16 months of follow-up. (Figures inside the bars are the mean abundance of the phylum for each group).

Figures S7. Linear size effect discriminant analysis (LDA LEfSe) of the gut bacterial community structure at species levels in 0 to 4 years old children who experienced (green), or 
did not experience (red), at least one malaria attack within 16 months of follow-up. Vertical bars represent the effect size for each taxon. The length of the bar represents the $\log 10$ transformed LDA score, indicated by vertical dotted lines.

Figure S8. Linear size effect discriminant analysis (LDA LEfSe) of the gut bacterial community structure at species levels, in 5 to 15 years old children who experienced (green), or did not experience (red), at least one malaria attack within 16 months of follow-up. Horizontal bars represent the effect size for each taxon. The length of the bar represents the $\log 10$ transformed LDA score, indicated by vertical dotted lines.

Figure S9. Linear size effect discriminant analysis (LDA LEfSe) of the gut bacterial community structure at species levels, in 0 to 4 years old children who developed (green), or did not develop (red), at least one asymptomatic Plasmodium parasitaemia episode within 16 months of follow-up. Vertical bars represent the effect size for each taxon. The length of the bar represents the $\log 10$ transformed LDA score, indicated by vertical dotted lines.

Figure S10. Linear size effect discriminant analysis (LDA LEfSe) of the gut bacterial community structure at species levels, in 5 to 15 years old children who developed (green), or did not develop (red), at least one asymptomatic Plasmodium parasitaemia episode within 16 months of follow-up. Horizontal bars represent the effect size for each taxon. The length of the bar represents the $\log 10$ transformed LDA score, indicated by vertical dotted lines.

Figure S11. Gut fungal community structure analysis. Scatter dot plot, median and range, of the Shannon H diversity index (Man Whitney test, $\mathrm{p}=0.66$ ) (a) and Box and Whiskers graph of the Chao- 1 richness index $(\mathrm{p}=0.9)(\mathrm{b})$ in children who experienced at least one malaria attack (crimson) or who did not (blue) within 16 months of follow-up. Scatter dot plot, median and range, of the Shannon $H$ diversity index (Man Whitney test, $\mathrm{p}=0.31$ ) (c) and Box and Whiskers graph of the Chao- 1 richness index $(p=0.87)(d)$ in children who developed at least one asymptomatic Plasmodium parasitaemia episode (red) or not (green) within 16 months of follow-up.

Figure S12. Gut fungal community structure. Relative abundance of phyla in children who experienced (yellow bars) or did not experience (blue bars) at least one malaria attack within 16 months of follow-up. (Figures inside the bars are the mean abundance of the phylum for each group). 
Figure S13. Gut fungal community structure. Relative abundance of phyla in children who developed (blue bars) or did not develop (yellow bars) at least one asymptomatic Plasmodium parasitaemia episode within 16 months of follow-up. (Figures inside the bars are the mean abundance of the phylum for each group).

Table S1. Malaria outcomes by age group within 16 months of follow-up.

Table S2. Logistic regression analysis of the association of age and gut bacterial and fungal community structure with the risk of at least one asymptomatic Plasmodium parasitaemia episode.

Table S3. Gut bacterial and fungal community structures according to the children's age groups.

Table S4. Gut bacterial and fungal community structure in children who experienced, or did not experience, at least one malaria attack within 16 months of follow-up.

Table S5. Gut bacterial and fungal community structure in children who developed, or did not develop, at least one asymptomatic Plasmodium parasitaemia episode within 16 months of follow-up. 